\title{
Estadísticas de mortalidad en México: Muertes registradas en el año 2002
}

D e acuerdo con las cifras del Instituto Nacional de Estadística, Geografía e Informática (INEGI), en 2002 se registraron en México 459687 defunciones, de las cuales, 2007 corresponden a extranjeros y mexicanos que residían en el extranjero pero fallecieron en el país, por lo que se excluyen del total para fines del análisis que a continuación se presenta.

De las personas que fallecieron y residían en el país, se tienen 457680 defunciones registradas en las 32 entidades federativas (16 676 defunciones más que el año anterior), 44.4\% correspondieron a mujeres y $55.6 \%$ a hombres. Se mantiene la tendencia decreciente de las muertes en los niños menores de cinco años de edad: en 2002, 95 de cada 1000 muertes ocurrieron en esa edad, aunque conviene observar que la variación estatal va desde 180 a 60, si no se consideran aquellas entidades en donde es alto el subregistro de muertes infantiles. Por otro lado, la mitad de las defunciones suceden después de los 69 años de edad en las mujeres, y después de los 61, en los hombres. En los cuadros II y III se presentan las variaciones de la edad de muerte entre las entidades federativas. Es importante mencionar que en algunos cuadros, además de la tasa cruda de mortalidad, se incluye la tasa estandarizada por edad; la razón es que esta última permite hacer comparaciones entre entidades federativas a pesar de que presentan diferente es- tructura de edades en la población. Para facilitar las comparaciones internacionales se decidió usar como población estándar la que recomienda la Organización Mundial de la Salud ${ }^{1}$ (ver nota metodológica).

En los cuadros V y VI se muestran las principales causas de mortalidad registradas en 2002 para mujeres y hombres a escala nacional. Se repite el mismo formato que se usó para el año 2000; es decir, las principales causas se presentan en un nivel de desagregación que incluso permite asociarlas con intervenciones en salud específicas, evitando las agrupaciones en la medida de lo posible. En este sentido, destaca la diabetes mellitus ocupando la primera causa de muerte en las mujeres $(14.6 \%)$ y la segunda en los hombres $(9.9 \%)$, seguida de la cardiopatía isquémica y de las enfermedades cerebrovasculares, las cuales aumentaron, tanto en números absolutos como en tasa.

Además, llama la atención que el orden se modificó en los hombres (cuadro VI), debido a la disminución de las defunciones por accidente de tráfico, las cuales descendieron del lugar 5 en 2001, al 9, y al aumento por agresiones (homicidios), -de ser la causa 8 en 2001 pasa a la 7 en 2002.

Los cuadros del VIII al XII incluyen las defunciones y las tasas estandarizadas, para mujeres y hombres, por entidad federativa, y algunas causas de muerte seleccionadas.
En los cuadros XIII y XIV se presentan indicadores seleccionados sobre la calidad de la información de la mortalidad. En la primera columna del cuadro XIII se muestra el porcentaje de muertes que ocurrieron antes del año 2001, pero que fueron registradas ese año y el INEGI las reporta en su estadística. En el ámbito nacional se registra alrededor de $2.1 \%$ de defunciones que ocurrieron en años anteriores, observándose que en algunas entidades el registro extemporáneo asciende hasta $9 \%{ }^{2}$

Tradicionalmente se ha considerado que el certificado de defunción debe ser llenado por el médico. En México, 98\% de ellos cumplen con este requisito; lo más recomendable es que el médico que trató al paciente sea quien extienda el certificado de defunción; sin embargo, eso no sucede con la frecuencia esperada en nuestro país. Si se descuentan las defunciones que por ley deben ser certificadas por los médicos legistas (aproximadamente 51703 defunciones por causas externas) sólo $31 \%$ son certificadas por el médico tratante; el resto las certifica otro médico que no conocía a la persona que falleció.

Cabe destacar que en el año 2001 las defunciones certificadas por el médico tratante eran $32.4 \%$, ahora son $28.2 \%$; en términos relativos al porcentaje es menor; sin embargo, en números absolutos ascendió de 126000 a 129 000. De la misma ma-

\footnotetext{
* Sección preparada por la Dirección General de Información en Salud. Secretaría de Salud, México.
} 
nera se utiliza el indicador de las defunciones que sucedieron en las unidades médicas, $45.2 \%$, en las que se presupone que el llenado de los certificados en dichos lugares fue de mejor calidad (cuadro XIII).

Otro criterio de calidad es la identificación de las variables de la persona que falleció; por ejemplo, la edad o la condición de derechohabiencia. En México, en 1890 defunciones de mayores de un año de edad no se especifica la edad, tampoco en 311 defunciones que sucedieron en menores de un año. Actualmente, uno de cada 11 certificados no especifica la variable derechohabiencia, en algunas entidades la relación asciende a uno de cada cinco.

La sección médica del certificado de defunción y la correcta definición de la causa de muerte son fundamentales. Conviene insistir en la importancia de escribir, en el certificado de defunción, la secuencia de los padecimientos que antecedieron a la muerte, con el fin de que el codificador pueda aplicar las reglas de codificación y el principio general que aparece en la Clasificación Estadística Internacional de Enfermedades y Problemas Relacionados con la Salud (CIE).

Desafortunadamente, en nuestro país todavía existen problemas en el llenado del certificado, los cuales repercuten en detrimento de la calidad de las estadísticas de mortalidad. En el cuadro XIV se presentan cinco indicadores que permiten evaluar la calidad de los certificados, en relación con la causa básica de muerte.

Del total de defunciones, $2 \%$ clasifican como Signos y síntomas mal definidos (CIE-10 R00-99), aunque en algunas entidades ascienden a $5.1 \%$. Llama la atención que, en términos absolutos, las defunciones cuya causa estuvo mal definida ascendió de 9195 en 2001, a 9359 en 2002. Otro criterio para medir la calidad de la causa de muerte se refiere a la precisión con la que una defunción por causa externa puede ser clasificada como intencional o accidental. La CIE-10 previene esta situación y establece un grupo de códigos para "Eventos de intención no determinada" (Y10-Y34). En 2002, en México, 5.4\% de las 52 mil muertes por causas externas no pueden ser clasificadas como accidentales o intencionales, y caen en este rubro, lo mismo sucede con las defunciones por tumores malignos de sitio no especificado.

Uno de los problemas típicos en la certificación de la muerte consiste en referir el mecanismo de ésta y no la causa o enfermedad que la desencadenó. Los mejores ejemplos en este sentido son las muertes por insuficiencia cardiaca (CIE-10 I50) que pueden tener un origen isquémico, reumático, asociarse a una cardiopatía hipertensiva o con una cardiopatía por enfermedad de Chagas; o la insuficiencia hepática (CIE-10 K72) que, en su caso, puede estar relacionada con cirrosis hepática, hepatitis crónica o, incluso, hematoma. Las últimas dos columnas del cuadro XIV dan cuenta de este problema al cual se podrían sumar la insuficiencia renal, la insuficiencia respiratoria y la ateroesclerosis inespecífica, entre otras. Lo paradójico del caso es que en aquellas entidades federativas en donde la proporción de insuficiencia cardiaca es alta (Tlaxcala, Oaxaca, Puebla), la mortalidad por cardiopatía isquémica es baja, lo cual genera la duda acerca de si la baja mortalidad es real o se asocia con la mala clasificación de las defunciones.

Por último, en el cuadro XV se presenta la mortalidad por ciertas afecciones perinatales, según entidad de residencia habitual, para los años 2000, 2001 y 2002.

En comparación con las defunciones registradas en el año 2001 se observan cambios en la estructura de causas y por edades, en la calidad y en la integridad de los datos. Aún se detectan problemas de subregistro en algunas entidades federativas, así como problemas en el manejo de causas de muerte; por lo anterior, es imperativo mantener los programas de capacitación para los médicos respecto del llenado del certificado de defunción, y con los codificadores, para aplicar las actualizaciones de la Clasificación Estadística Internacional de Enfermedades y Problemas Relacionados con la Salud.

\section{Nota metodológica}

Uno de los procedimientos utilizados en el cálculo y presentación de tasas es el de estandarización. Con ello, se controla el efecto de la variación en la estructura de edades de una población, y tiene la finalidad de elaborar indicadores resumen, comparables en diferentes contextos; también sirve para analizar su evolución en el tiempo.

El procedimiento de estandarización consiste en calcular una tasa resumen a partir de la suma ponderada de las tasas observadas, por grupo de edad de la población de estudio.

Los ponderadores utilizados se corresponden con los valores derivados de la distribución porcentual, por grupos de edad, de la población tipo. Dado que la selección de la población tipo es arbitraria, en el caso que nos ocupa se consideró utilizar, para efectos de comparación internacional, la estructura reportada por la Organización Mundial de la Salud, que es el resultado de promediar las estructuras por edad de la población mundial esperadas para los años 2000 y 2025.

\section{Referencias}

\section{W orld Health 0 rganization. A ge}

standarization of rates: A new W HO standard. Ginebra:W HO, 2000. (GPE D iscussion Paper Series N 0. 31).

2. Secretaría de Salud. Dirección General de Información en Salud. Tendencia de la mortalidad en México 1990-2000. Muertes ocurridas vs muertes registras. En prensa. 


\section{Cuadro I \\ MoRTALIDAD General (CRUDA Y ESTANDARIZADA POR EDAD) POR SEXO, SEGÚN ENTIDAD FEDERATIVA DE RESIDENCIA HABITUAL. MéXICO, 2002}

\begin{tabular}{|c|c|c|c|c|c|c|c|c|c|c|c|}
\hline \multirow[b]{2}{*}{ Entidad federativa } & \multicolumn{3}{|c|}{ Total } & \multicolumn{3}{|c|}{ M ujeres } & \multicolumn{3}{|c|}{ Hombres } & \multirow[b]{2}{*}{$N E$} & \multirow[b]{2}{*}{ ISM } \\
\hline & Defunciones & Tasa* & Estandarizada ${ }^{\ddagger}$ & Defunciones & Tasa* & Estandarizada ${ }^{\ddagger}$ & Defunciones & Tasa* & Estandarizada ${ }^{\ddagger}$ & & \\
\hline Nacional & 457680 & 4.4 & 6.3 & 203252 & 3.9 & 5.3 & 254112 & 5.0 & 7.5 & 316 & 125.0 \\
\hline A guascalientes & 4301 & 4.3 & 6.7 & 2018 & 4.0 & 5.8 & 2277 & 4.7 & 7.8 & 6 & 112.8 \\
\hline Baja California & 11365 & 4.2 & 7.0 & 4407 & 3.3 & 5.6 & 6954 & 5.1 & 8.4 & 4 & 157.8 \\
\hline Baja California Sur & 1785 & 3.9 & 6.4 & 706 & 3.1 & 5.0 & 1079 & 4.5 & 7.8 & 0 & 152.8 \\
\hline Campeche & 2750 & 3.7 & 6.0 & 1121 & 3.1 & 4.9 & 1629 & 4.4 & 7.2 & 0 & 145.3 \\
\hline Coahuila & 11030 & 4.5 & 6.9 & 4989 & 4.1 & 6.0 & 6036 & 4.9 & 7.9 & 5 & 121.0 \\
\hline Colima & 2512 & 4.4 & 6.2 & 1052 & 3.7 & 4.9 & 1459 & 5.1 & 7.5 & 1 & 138.7 \\
\hline Chiapas & 16424 & 3.9 & 6.9 & 7071 & 3.4 & 5.8 & 9333 & 4.4 & 8.2 & 20 & 132.0 \\
\hline Chihuahua & 16494 & 5.1 & 7.5 & 6910 & 4.3 & 6.1 & 9540 & 5.8 & 9.0 & 44 & 138.1 \\
\hline Distrito Federal & 46984 & 5.3 & 6.3 & 22988 & 5.1 & 5.6 & 23987 & 5.6 & 7.0 & 9 & 104.3 \\
\hline Durango & 6308 & 4.1 & 5.9 & 2620 & 3.4 & 4.7 & 3675 & 4.8 & 7.3 & 13 & 140.3 \\
\hline Guanajuato & 22285 & 4.5 & 6.5 & 10323 & 4.1 & 5.5 & 11958 & 5.0 & 7.6 & 4 & 115.8 \\
\hline Guerrero & 10882 & 3.4 & 4.8 & 4704 & 2.9 & 3.9 & 6169 & 3.9 & 5.8 & 9 & 131.1 \\
\hline Hidalgo & 10151 & 4.4 & 6.0 & 4402 & 3.7 & 4.7 & 5748 & 5.0 & 7.4 & 1 & 130.6 \\
\hline Jalisco & 32339 & 4.9 & 6.4 & 14755 & 4.4 & 5.5 & 17568 & 5.3 & 7.5 & 16 & 119.1 \\
\hline México & 54643 & 3.9 & 6.4 & 24458 & 3.5 & 5.5 & 30131 & 4.3 & 7.2 & 54 & 123.2 \\
\hline Michoacán & 19084 & 4.6 & 6.0 & 8550 & 4.0 & 5.0 & 10519 & 5.1 & 7.1 & 15 & 123.0 \\
\hline Morelos & 7213 & 4.3 & 5.9 & 3221 & 3.9 & 5.0 & 3983 & 4.8 & 6.8 & 9 & 123.7 \\
\hline $\mathrm{N}$ ayarit & 4508 & 4.6 & 6.0 & 1864 & 3.9 & 4.7 & 2642 & 5.4 & 7.3 & 2 & 141.7 \\
\hline N uevo León & 16849 & 4.2 & 6.1 & 7521 & 3.7 & 5.1 & 9321 & 4.6 & 7.2 & 7 & 123.9 \\
\hline 0 axaca & 17957 & 4.9 & 6.4 & 8070 & 4.4 & 5.3 & 9872 & 5.5 & 7.6 & 15 & 122.3 \\
\hline Puebla & 26760 & 5.0 & 6.7 & 12016 & 4.4 & 5.6 & 14738 & 5.6 & 8.1 & 6 & 122.7 \\
\hline Q uerétaro & 6188 & 4.1 & 6.5 & 2681 & 3.5 & 5.3 & 3502 & 4.7 & 7.8 & 5 & 130.6 \\
\hline Q uintana Roo & 2552 & 2.6 & 5.9 & 954 & 2.0 & 4.6 & 1593 & 3.2 & 7.1 & 5 & 167.0 \\
\hline San Luis Potosí & 10535 & 4.4 & 6.0 & 4739 & 3.9 & 4.9 & 5786 & 4.9 & 7.1 & 10 & 122.1 \\
\hline Sinaloa & 10798 & 4.0 & 5.8 & 4395 & 3.3 & 4.6 & 6395 & 4.7 & 7.0 & 8 & 145.5 \\
\hline Sonora & 10995 & 4.6 & 6.9 & 4486 & 3.8 & 5.5 & 6509 & 5.4 & 8.3 & 0 & 145.1 \\
\hline Tabasco & 7662 & 3.8 & 6.5 & 3122 & 3.1 & 5.2 & 4536 & 4.5 & 7.8 & 4 & 145.3 \\
\hline Tamaulipas & 11970 & 4.0 & 5.8 & 5172 & 3.5 & 4.8 & 6790 & 4.5 & 6.9 & 8 & 131.3 \\
\hline Tlaxcala & 4633 & 4.5 & 6.3 & 2072 & 4.0 & 5.3 & 2561 & 5.1 & 7.6 & 0 & 123.6 \\
\hline Veracruz & 34216 & 4.7 & 6.3 & 14855 & 4.1 & 5.2 & 19357 & 5.4 & 7.6 & 4 & 130.3 \\
\hline Yucatán & 8681 & 5.0 & 6.6 & 3948 & 4.5 & 5.5 & 4732 & 5.5 & 7.8 & 1 & 119.9 \\
\hline Zacatecas & 6826 & 4.8 & 6.1 & 3062 & 4.2 & 5.1 & 3733 & 5.4 & 7.3 & 31 & 121.9 \\
\hline
\end{tabular}

* Tasa por 1000 habitantes, según sexo

₹ Tasa por 1000 habitantes estandarizada por el método directo usando la población mundial estándar Word Health O rganization.Age Standardization of rates:A new W HO standard

Los totales no incluyen 2007 defunciones de mexicanos que residían en el extranjero

ISM = índice de sobremortalidad masculina

Fuente:Instituto N acional de Estadística, G eografía e Informática/Secretaría de Salud.D irección General de Información en Salud. Base de datos de defunciones 2002. México, 2004 


\section{Cuadro II}

Distribución de LAS defunciones de MUJeRES POR GRUPOS DE EDAD, SEgún ENTIDAd FEDERATIVA DE RESIDENCIA HABITUAL. MÉXICO, 2002

\begin{tabular}{|c|c|c|c|c|c|c|c|c|c|c|c|}
\hline Entidad federativa & Total & $0-4$ & $5-14$ & $15-24$ & $25-34$ & $35-44$ & $45-54$ & $55-64$ & $65-74$ & $75 y+$ & NE \\
\hline$N$ acional & 203252 & 9.2 & 1.4 & 2.5 & 3.1 & 4.7 & 8.0 & 12.5 & 18.2 & 40.2 & 0.3 \\
\hline A guascalientes & 2018 & 9.5 & 1.6 & 2.0 & 2.9 & 4.4 & 7.3 & 11.3 & 17.5 & 43.1 & 0.4 \\
\hline Baja California & 4407 & 10.9 & 1.7 & 3.2 & 4.3 & 6.4 & 8.5 & 13.4 & 18.4 & 33.0 & 0.2 \\
\hline Baja C alifornia Sur & 706 & 10.2 & 1.8 & 2.8 & 5.1 & 5.9 & 5.9 & 12.2 & 18.8 & 36.8 & 0.3 \\
\hline Campeche & 1121 & 7.7 & 0.8 & 3.5 & 3.5 & 4.8 & 8.4 & 14.5 & 20.0 & 36.2 & 0.7 \\
\hline Coahuila & 4989 & 5.4 & 1.0 & 1.9 & 2.6 & 4.5 & 9.1 & 15.7 & 21.0 & 38.1 & 0.6 \\
\hline Colima & 1052 & 5.9 & 1.5 & 2.3 & 1.5 & 4.2 & 9.7 & 10.6 & 16.3 & 46.8 & 1.2 \\
\hline Chiapas & 7071 & 13.0 & 2.5 & 4.5 & 4.8 & 6.9 & 9.7 & 12.9 & 15.7 & 29.3 & 0.6 \\
\hline Chihuahua & 6910 & 9.3 & 1.5 & 2.9 & 3.3 & 4.9 & 8.8 & 13.2 & 20.1 & 35.2 & 0.8 \\
\hline Distrito Federal & 22988 & 6.1 & 0.7 & 1.6 & 2.4 & 4.0 & 7.8 & 12.2 & 19.6 & 45.6 & 0.0 \\
\hline Durango & 2620 & 6.0 & 1.2 & 2.4 & 3.0 & 3.9 & 7.0 & 13.4 & 21.0 & 41.9 & 0.3 \\
\hline Guanajuato & 10323 & 10.6 & 1.4 & 2.1 & 2.6 & 4.3 & 6.8 & 11.3 & 16.6 & 43.5 & 0.7 \\
\hline Guerrero & 4704 & 7.2 & 1.7 & 3.4 & 4.7 & 6.2 & 8.4 & 13.0 & 19.2 & 35.7 & 0.5 \\
\hline Hidalgo & 4402 & 9.0 & 1.5 & 2.7 & 3.0 & 5.0 & 8.1 & 11.7 & 16.7 & 41.8 & 0.7 \\
\hline Jalisco & 14755 & 7.6 & 1.3 & 2.4 & 2.4 & 4.0 & 6.9 & 12.0 & 17.2 & 45.9 & 0.1 \\
\hline México & 24458 & 13.7 & 1.5 & 2.8 & 3.6 & 5.3 & 9.1 & 13.1 & 17.3 & 33.7 & 0.1 \\
\hline Michoacán & 8550 & 7.0 & 1.5 & 2.3 & 2.5 & 3.9 & 7.2 & 11.0 & 18.7 & 45.6 & 0.3 \\
\hline Morelos & 3221 & 7.9 & 1.1 & 2.7 & 2.5 & 5.2 & 7.2 & 12.3 & 18.8 & 41.9 & 0.6 \\
\hline $\mathrm{N}$ ayarit & 1864 & 5.2 & 1.6 & 2.5 & 3.0 & 4.2 & 7.7 & 11.6 & 19.6 & 44.3 & 0.4 \\
\hline Nuevo León & 7521 & 6.2 & 1.0 & 1.6 & 2.5 & 4.2 & 7.8 & 13.7 & 20.2 & 41.9 & 0.8 \\
\hline 0 axaca & 8070 & 10.0 & 1.8 & 2.7 & 3.1 & 4.7 & 7.8 & 12.0 & 18.3 & 39.3 & 0.4 \\
\hline Puebla & 12016 & 14.7 & 1.7 & 2.4 & 3.0 & 4.5 & 7.1 & 11.1 & 16.6 & 38.6 & 0.1 \\
\hline Q uerétaro & 2681 & 12.6 & 1.9 & 2.4 & 3.5 & 4.8 & 7.8 & 11.9 & 14.7 & 39.9 & 0.5 \\
\hline Q uintana Roo & 954 & 18.0 & 1.8 & 4.5 & 5.2 & 7.5 & 9.0 & 12.6 & 15.7 & 24.4 & 1.2 \\
\hline San Luis Potosí & 4739 & 9.5 & 1.5 & 2.4 & 2.5 & 3.7 & 7.9 & 10.8 & 17.1 & 44.5 & 0.2 \\
\hline Sinaloa & 4395 & 4.3 & 1.1 & 2.3 & 3.0 & 4.5 & 6.8 & 12.9 & 19.3 & 45.3 & 0.4 \\
\hline Sonora & 4486 & 8.4 & 1.4 & 2.8 & 2.9 & 3.7 & 7.7 & 14.0 & 19.6 & 39.4 & 0.2 \\
\hline Tabasco & 3122 & 11.0 & 2.4 & 2.9 & 4.5 & 5.6 & 9.3 & 12.4 & 17.4 & 34.2 & 0.2 \\
\hline Tamaulipas & 5172 & 6.1 & 1.1 & 1.8 & 3.2 & 5.1 & 8.9 & 13.5 & 20.7 & 39.1 & 0.5 \\
\hline Tlaxcala & 2072 & 13.1 & 1.4 & 2.8 & 3.1 & 4.0 & 7.9 & 10.7 & 15.5 & 41.2 & 0.2 \\
\hline Veracruz & 14855 & 7.8 & 1.5 & 2.5 & 3.2 & 5.1 & 8.4 & 13.4 & 19.2 & 38.5 & 0.4 \\
\hline Yucatán & 3948 & 6.6 & 1.3 & 1.6 & 2.4 & 3.9 & 6.9 & 12.3 & 17.7 & 47.0 & 0.4 \\
\hline Zacatecas & 3062 & 9.3 & 1.2 & 2.3 & 2.9 & 3.7 & 6.5 & 10.9 & 15.8 & 47.0 & 0.4 \\
\hline
\end{tabular}

Los totales no incluyen 594 defunciones de mexicanas que residían en el extranjero $\mathrm{NE}=$ no especificado

Fuente: Instituto Nacional de Estadística, Geografía e Informática/Secretaría de Salud. Dirección General de Información en Salud. Base de datos de defunciones 2002. México, 2004 


\section{Cuadro III \\ Distribución DE LAS DEFUNCIONES DE HOMBRES POR GRUPOS DE EDAD, SEGÚN ENTIDAD FEDERATIVA DE RESIDENCIA habITUAL. MÉXICO, 2002}

\begin{tabular}{|c|c|c|c|c|c|c|c|c|c|c|c|}
\hline Entidad federativa & Total & $0-4$ & 5-14 & $15-24$ & $25-34$ & $35-44$ & $45-54$ & $55-64$ & $65-74$ & $75 y+$ & $N E$ \\
\hline $\mathrm{N}$ acional & 254112 & 9.6 & 1.6 & 4.8 & 6.5 & 7.9 & 10.0 & 13.2 & 17.1 & 28.6 & 0.6 \\
\hline Aguascalientes & 2277 & 11.8 & 2.0 & 4.3 & 5.8 & 7.4 & 8.7 & 12.7 & 16.0 & 30.5 & 0.8 \\
\hline Baja California & 6954 & 9.3 & 1.3 & 5.5 & 9.9 & 11.5 & 12.1 & 12.8 & 16.2 & 20.1 & 1.3 \\
\hline Baja California Sur & 1079 & 9.5 & 1.6 & 4.5 & 9.2 & 7.8 & 10.0 & 12.9 & 17.5 & 25.8 & 1.2 \\
\hline Campeche & 1629 & 8.1 & 1.7 & 6.0 & 7.2 & 7.7 & 10.1 & 12.3 & 16.4 & 29.5 & 1.0 \\
\hline Coahuila & 6036 & 6.2 & 1.2 & 4.4 & 5.4 & 6.2 & 10.1 & 15.2 & 20.3 & 29.9 & 0.9 \\
\hline Colima & 1459 & 6.4 & 1.6 & 4.9 & 8.0 & 6.4 & 9.7 & 12.3 & 16.4 & 32.3 & 1.9 \\
\hline Chiapas & 9333 & 11.9 & 2.5 & 6.7 & 8.7 & 9.0 & 9.9 & 12.5 & 14.1 & 23.3 & 1.4 \\
\hline Chihuahua & 9540 & 8.5 & 1.5 & 5.8 & 7.5 & 9.1 & 9.6 & 13.5 & 17.4 & 25.9 & 1.2 \\
\hline Distrito Federal & 23987 & 7.5 & 0.9 & 4.0 & 6.3 & 8.0 & 10.7 & 14.6 & 18.5 & 29.4 & 0.0 \\
\hline Durango & 3675 & 7.3 & 1.2 & 5.2 & 6.0 & 5.9 & 9.2 & 12.7 & 20.2 & 31.8 & 0.5 \\
\hline Guanajuato & 11958 & 12.4 & 2.1 & 5.0 & 5.2 & 6.7 & 8.3 & 11.7 & 16.0 & 32.1 & 0.6 \\
\hline Guerrero & 6169 & 7.6 & 1.8 & 5.7 & 9.0 & 9.0 & 10.1 & 12.0 & 17.5 & 26.5 & 0.6 \\
\hline Hidalgo & 5748 & 9.8 & 1.9 & 4.2 & 5.6 & 7.1 & 10.9 & 14.8 & 17.3 & 28.0 & 0.5 \\
\hline Jalisco & 17568 & 8.0 & 1.7 & 5.1 & 5.9 & 6.9 & 9.4 & 12.3 & 16.1 & 34.3 & 0.3 \\
\hline México & 30131 & 14.1 & 1.6 & 5.4 & 7.1 & 9.1 & 11.6 & 14.2 & 15.5 & 21.2 & 0.2 \\
\hline Michoacán & 10519 & 7.7 & 1.9 & 5.6 & 6.1 & 7.5 & 8.7 & 11.0 & 16.5 & 34.5 & 0.5 \\
\hline Morelos & 3983 & 8.6 & 1.3 & 4.0 & 6.3 & 7.4 & 10.2 & 13.2 & 17.4 & 30.4 & 1.2 \\
\hline$N$ ayarit & 2642 & 5.6 & 1.6 & 5.7 & 6.5 & 6.7 & 8.7 & 11.5 & 17.9 & 35.0 & 0.7 \\
\hline N uevo León & 9321 & 6.8 & 1.1 & 3.5 & 4.8 & 6.5 & 9.7 & 15.9 & 20.8 & 30.3 & 0.6 \\
\hline 0 axaca & 9872 & 10.3 & 2.4 & 4.6 & 6.4 & 8.5 & 9.9 & 12.4 & 15.3 & 29.6 & 0.6 \\
\hline Puebla & 14738 & 16.0 & 1.9 & 3.9 & 5.8 & 7.9 & 10.0 & 12.3 & 15.5 & 26.3 & 0.3 \\
\hline Q uerétaro & 3502 & 11.5 & 2.2 & 5.2 & 6.7 & 8.5 & 10.7 & 12.6 & 14.7 & 27.4 & 0.6 \\
\hline Q uintana Roo & 1593 & 14.0 & 1.5 & 6.7 & 9.7 & 11.3 & 11.8 & 12.6 & 11.2 & 17.6 & 3.5 \\
\hline San Luis Potosí & 5786 & 9.9 & 2.1 & 4.9 & 4.6 & 6.4 & 8.3 & 12.0 & 16.9 & 34.6 & 0.2 \\
\hline Sinaloa & 6395 & 4.5 & 1.3 & 5.0 & 7.4 & 7.4 & 8.5 & 13.7 & 20.1 & 31.6 & 0.4 \\
\hline Sonora & 6509 & 7.3 & 1.4 & 4.2 & 5.7 & 7.1 & 9.4 & 14.8 & 20.6 & 28.9 & 0.6 \\
\hline Tabasco & 4536 & 10.3 & 2.5 & 7.1 & 8.6 & 8.6 & 10.3 & 11.3 & 15.0 & 25.7 & 0.7 \\
\hline Tamaulipas & 6790 & 5.4 & 1.1 & 3.9 & 6.2 & 7.5 & 10.1 & 15.6 & 19.7 & 29.5 & 0.9 \\
\hline Tlaxcala & 2561 & 14.0 & 2.1 & 5.0 & 6.3 & 6.8 & 7.8 & 11.4 & 15.2 & 30.9 & 0.5 \\
\hline Veracruz & 19357 & 7.9 & 1.5 & 4.1 & 6.1 & 8.6 & 10.9 & 14.3 & 18.1 & 27.6 & 0.8 \\
\hline Yucatán & 4732 & 7.2 & 1.2 & 3.3 & 5.6 & 6.8 & 10.0 & 11.2 & 16.2 & 38.1 & 0.5 \\
\hline Zacatecas & 3733 & 8.4 & 2.0 & 5.6 & 4.8 & 5.5 & 7.1 & 9.6 & 18.2 & 38.3 & 0.5 \\
\hline
\end{tabular}

Los totales no incluyen 1410 defunciones de mexicanos que residían en el extranjero $\mathrm{NE}=$ no especificado

Fuente: Instituto Nacional de Estadística, Geografía e Informática/Secretaría de Salud. Dirección General de Información en Salud. Base de datos de defunciones 2002. México, 2004 


\section{Cuadro IV \\ TASA DE MORTALIDAD (ESTANDARIZADA POR EDAD) POR GRANDES GRUPOS DE CAUSAS, SEgún SEXo Y ENTIDAd federativa. México, 2002}

\begin{tabular}{|c|c|c|c|c|c|c|c|c|c|}
\hline \multirow[b]{2}{*}{ Entidad federativa } & \multicolumn{3}{|c|}{ Transmisibles, nutricionales y de la reproducción* } & \multicolumn{3}{|c|}{ No transmisibles ${ }^{\ddagger}$} & \multicolumn{3}{|c|}{ Lesiones $^{\S}$} \\
\hline & Total & M ujeres & Hombres & Total & Mujeres & Hombres & Total & Mujeres & Hombres \\
\hline$N$ acional & 75.6 & 65.9 & 85.8 & 487.1 & 430.1 & 552.1 & 56.9 & 23.5 & 92.2 \\
\hline Aguascalientes & 62.1 & 55.8 & 68.0 & 538.8 & 485.7 & 602.3 & 62.4 & 29.8 & 100.4 \\
\hline Baja California & 78.9 & 56.8 & 100.9 & 534.1 & 463.4 & 606.9 & 70.9 & 28.2 & 113.0 \\
\hline Baja California Sur & 66.5 & 56.9 & 76.9 & 504.1 & 404.5 & 613.6 & 59.3 & 28.6 & 88.1 \\
\hline Campeche & 72.7 & 59.3 & 86.7 & 449.1 & 399.1 & 504.6 & 62.4 & 20.9 & 104.3 \\
\hline Coahuila & 63.3 & 52.2 & 75.5 & 557.2 & 504.0 & 617.8 & 49.9 & 23.3 & 76.5 \\
\hline Colima & 65.2 & 57.5 & 72.5 & 486.4 & 409.9 & 570.5 & 56.5 & 20.0 & 94.9 \\
\hline Chiapas & 110.5 & 96.8 & 125.9 & 458.4 & 409.4 & 514.4 & 65.9 & 23.2 & 110.1 \\
\hline Chihuahua & 82.8 & 71.3 & 94.1 & 562.5 & 490.8 & 642.6 & 83.1 & 33.8 & 132.5 \\
\hline Distrito Federal & 67.8 & 61.1 & 74.4 & 508.8 & 471.5 & 550.2 & 45.1 & 20.6 & 71.6 \\
\hline Durango & 52.5 & 42.0 & 63.3 & 461.8 & 392.3 & 538.8 & 61.5 & 23.9 & 101.0 \\
\hline Guanajuato & 78.3 & 70.2 & 86.8 & 507.1 & 450.5 & 576.2 & 54.6 & 23.2 & 90.2 \\
\hline Guerrero & 62.0 & 53.9 & 71.0 & 333.2 & 296.6 & 376.6 & 72.8 & 30.1 & 118.5 \\
\hline Hidalgo & 68.1 & 58.5 & 79.0 & 470.6 & 390.1 & 564.7 & 50.5 & 18.6 & 85.8 \\
\hline Jalisco & 72.5 & 63.5 & 81.9 & 508.1 & 455.3 & 568.3 & 58.4 & 26.4 & 91.9 \\
\hline México & 80.3 & 72.6 & 87.8 & 499.3 & 455.4 & 546.4 & 52.2 & 23.6 & 82.0 \\
\hline Michoacán & 62.3 & 54.2 & 70.8 & 456.2 & 406.6 & 514.6 & 70.1 & 29.0 & 115.7 \\
\hline Morelos & 73.7 & 64.1 & 84.0 & 454.5 & 408.1 & 505.4 & 50.2 & 18.0 & 83.8 \\
\hline $\mathrm{N}$ ayarit & 65.0 & 54.1 & 76.8 & 448.8 & 384.3 & 522.4 & 73.8 & 24.9 & 123.0 \\
\hline Nuevo León & 55.5 & 43.4 & 69.3 & 510.0 & 446.1 & 583.1 & 40.1 & 18.3 & 62.2 \\
\hline 0 axaca & 109.5 & 100.2 & 119.8 & 431.5 & 379.3 & 492.4 & 68.0 & 24.6 & 116.1 \\
\hline Puebla & 104.4 & 92.9 & 116.6 & 487.7 & 414.7 & 574.1 & 54.2 & 24.2 & 86.9 \\
\hline Querétaro & 78.1 & 71.1 & 85.2 & 499.9 & 418.6 & 594.2 & 58.8 & 27.6 & 91.7 \\
\hline Q uintana Roo & 59.7 & 46.8 & 72.7 & 440.6 & 376.2 & 507.7 & 65.3 & 20.2 & 109.6 \\
\hline San Luis Potosí & 76.2 & 67.8 & 86.0 & 445.3 & 388.2 & 514.2 & 56.1 & 21.1 & 94.4 \\
\hline Sinaloa & 50.6 & 44.9 & 55.9 & 453.5 & 385.1 & 526.1 & 63.9 & 21.9 & 105.2 \\
\hline Sonora & 74.8 & 65.1 & 84.7 & 542.7 & 448.8 & 644.3 & 58.7 & 23.1 & 93.8 \\
\hline Tabasco & 74.2 & 60.2 & 89.0 & 493.7 & 431.0 & 565.0 & 66.1 & 20.8 & 112.4 \\
\hline Tamaulipas & 48.4 & 40.3 & 57.3 & 478.4 & 415.4 & 548.9 & 48.1 & 20.3 & 76.1 \\
\hline Tlaxcala & 91.8 & 81.8 & 102.6 & 461.1 & 406.5 & 529.1 & 65.2 & 28.8 & 104.2 \\
\hline Veracruz & 79.1 & 66.1 & 93.1 & 490.6 & 418.9 & 573.2 & 49.7 & 18.6 & 83.0 \\
\hline Yucatán & 78.8 & 66.8 & 93.0 & 496.3 & 441.7 & 562.9 & 53.4 & 18.6 & 90.3 \\
\hline Zacatecas & 69.8 & 64.8 & 74.6 & 458.9 & 399.8 & 526.9 & 68.4 & 28.4 & 112.5 \\
\hline
\end{tabular}

* Incluye enfermedades infecciosas y parasitarias, muertes maternas, perinatales y deficiencias nutricias A 00-B99,G 00-G 04,N 70-N 73,J00-J06,J10-J18,J20122,H65-H66,0 00-0 99,P00-P96,E00-E02,E40-E46,E50,D50-D 64 (CIE 10a\# revisión)

₹ C 00-D 48,D 65-D 89,E03-E34,E51-F99,G 06-H61,H68-I99,J30-N 64,N 75-M99,Q 00-Q 99 (CIE 10a revisión)

$\S$ V01-Y89 (CIE 10a revisión)

* CIE 10: Clasificación Estadística Internacional de Enfermedades y Problemas Relacionados con la Salud. 10a revisión

Tasa por 100000 habitantes estandarizada por el método directo usando la población mundial estándar (ver nota metodológica) Los totales no incluyen defunciones de mexicanos que residían en el extranjero

Fuente: Instituto Nacional de Estadística, Geografía e Informática/Secretaría de Salud. Dirección General de Información en Salud. Base de datos de defunciones 2002. México, 2004 


\section{Cuadro V}

Principales causas de mortalidad en mujeres. México, 2002

Número de orden

Causa

Clave CIE* 10a Rev.

Defunciones $\quad$ Tasa $^{\ddagger} \quad \%$

Total

A00-Y 98

$203252 \quad 392.6 \quad 100.0$

\begin{tabular}{ll}
1 & Diabetes mellitus \\
\hline 2 & Enfermedades isquémicas del corazón \\
\hline 3 & Enfermedad cerebrovascular \\
\hline 4 & Ciertas afecciones originadas en el periodo perinatal \\
\hline 5 & Enfermedad pulmonar obstructiva crónica
\end{tabular}

E10-E14 $29673 \quad 57.3 \quad 14.6$

120-125 $21650 \quad 41.8 \quad 10.7$

$160-169 \quad 14145 \quad 27.3 \quad 7.0$

\begin{tabular}{|c|c|c|c|c|c|}
\hline 5 & rónica & 67 & 74 & 4.1 & 6 \\
\hline 6 & Infecciones respiratorias agudas bajas & J10-J18, J20-J22 & 6425 & 12.4 & 3.2 \\
\hline 7 & Enfermedades hipertensivas & $|10-| 15$ & 6282 & 12.1 & 3.1 \\
\hline 8 & Cirrosis y otras enfermedades crónicas del hígado & $\mathrm{K} 70, \mathrm{~K} 72.1, \mathrm{~K} 73, \mathrm{~K} 74, \mathrm{~K} 76$ & 6177 & 11.9 & 3.0 \\
\hline 9 & $\mathrm{~N}$ efritis y nefrosis & N O0-N 19 & 4771 & 9.2 & 2.4 \\
\hline 10 & Desnutrición calórico proteica & E40-E46 & 4613 & 8.9 & 2.3 \\
\hline 11 & Tumor maligno del cuello del útero & $\mathrm{C} 53$ & 4323 & 8.4 & 2.1 \\
\hline 12 & Tumor maligno de la mama & $\mathrm{C} 50$ & 3837 & 7.4 & 1.9 \\
\hline 13 & Tumor maligno del estómago & C16 & 2367 & 4.6 & 1.2 \\
\hline 14 & Tumor maligno del hígado & $\mathrm{C} 22$ & 2362 & 4.6 & 1.2 \\
\hline 15 & Enfermedades infecciosas intestinales & A00-A09 & 2324 & 4.5 & 1.1 \\
\hline 16 & Tumor maligno de tráquea, bronquios y pulmón & C $33-C 34$ & 2140 & 4.1 & 1.1 \\
\hline 17 & Accidentes de vehículo de motor (tránsito) & $\$$ & 2029 & 3.9 & 1.0 \\
\hline 18 & Malformaciones congénitas del corazón & Q 20-Q 24 & 1693 & 3.3 & 0.8 \\
\hline 19 & Anemia & D50-D64 & 1687 & 3.3 & 0.8 \\
\hline \multirow[t]{3}{*}{20} & Tumor maligno del páncreas & $\mathrm{C} 25$ & 1551 & 3.0 & 0.8 \\
\hline & Causas mal definidas & R00-R99 & 4647 & 9.0 & 2.3 \\
\hline & Las demás & 65476 & 126.5 & 32.2 & \\
\hline
\end{tabular}

* CIE 10: Clasificación Estadística Internacional de Enfermedades y Problemas Relacionados con la Salud. 10a revisión

₹ Tasa por 100000 mujeres

§ V02-V04 (.1, .9),V09.2-V09.3,V09.9,V12-V14 (.3-.9),V19.4-V19.6,V20-V28 (.3-.9),V29-V79 (.4-.9),V80.3-V80.5,V81.1,V82.1, V83-V86 (.0-.3),V87.0-V87.8, $\mathrm{V} 89.2, \mathrm{~V} 89.9, \mathrm{Y} 85.0$

Los totales no incluyen las defunciones de residentes en el extranjero

Fuente: Instituto Nacional de Estadística, Geografía e Informática/Secretaría de Salud. Dirección General de Información en Salud. Base de datos de defunciones 2002. México, 2004 


\section{Principales causas de mortalidad en hombres. México, 2002}

\begin{tabular}{ll}
1 & Enfermedades isquémicas del corazón \\
\hline 2 & Diabetes mellitus \\
\hline 3 & Cirrosis y otras enfermedades crónicas del hígado \\
\hline 4 & Enfermedad cerebrovascular
\end{tabular}

$120-125$

E10-E14

$26618 \quad 51.9 \quad 10.5$

\begin{tabular}{ll}
\hline 5 & Ciertas afecciones originadas en el periodo perinatal \\
\hline 6 & Enfermedad pulmonar obstructiva crónica \\
\hline 7 & Agresiones (homicidios)
\end{tabular}

$25141 \quad 49.0 \quad 9.9$

\begin{tabular}{rlll}
\hline 8 & Infecciones respiratorias agudas bajas & J10-J18, J20-|22 & 7 \\
\hline 9 & Accidentes de vehículo de motor (tránsito) & $\$$ & 7 \\
\hline 10 & N efritis y nefrosis & N 00-N 19 & 5 \\
\hline 11 & Tumor maligno de tráquea, bronquios y pulmón & C 33-C 34 & 453 \\
\hline 12 & Enfermedades hipertensivas & I10-I15 & 4
\end{tabular}

\begin{tabular}{lll}
\hline 13 & Desnutrición calórico proteica & E40-E46 \\
\hline 14 & Tumor maligno de la próstata & C 61
\end{tabular}

\begin{tabular}{lll}
\hline 15 & Peatón lesionado en accidente de vehículo de motor & V02-V04 (.1, .9),V09.2-V09.3,V09.9 \\
\hline 16 & Uso de alcohol & F10, G31.2
\end{tabular}

\begin{tabular}{llll}
\hline 17 & VIH/SIDA & B20-B24 & 374 \\
\hline 18 & Lesiones autoinfligidas intencionalmente (suicidios) & X60-X 84,Y87.0 & 3 \\
\hline 19 & Tumor maligno del estómago & C 16 & 274 \\
\hline
\end{tabular}

\begin{tabular}{lll}
2349 & 5.4 & 1.1 \\
\hline & 4.6 & 0.9
\end{tabular}

Causas mal definidas

Las demás
R00-R99

79436

$\begin{array}{lll}4694 & 9.2 & 1.9\end{array}$

$154.9 \quad 31.3$

* CIE 10: Clasificación Estadística Internacional de Enfermedades y Problemas Relacionados con la Salud. 10a revisión

* Tasa por 100000 habitantes

§ V02-V04 (.1, .9),V09.2-V09.3,V09.9,V12-V14 (.3-.9),V19.4-V19.6,V20-V28 (.3-.9),V29-V79 (.4-.9),V80.3-V80.5,V81.1,V82.1, V83-V86 (.0-.3),V87.0-V87.8, $\mathrm{V} 89.2, \mathrm{~V} 89.9, \mathrm{Y} 85.0$

Los totales no incluyen las defunciones de residentes en el extranjero

Fuente: Instituto Nacional de Estadística, Geografía e Informática/Secretaría de Salud. Dirección General de Información en Salud. Base de datos de defunciones 2002. México, 2004 


\section{Cuadro VII \\ MORTALIDAD EN MUJERES (ESTANDARIZADA POR EDAD) POR ENFERMEDADES TRANSMISIBLES, PERINATALES

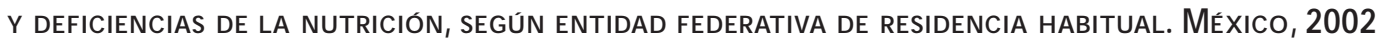

\begin{tabular}{|c|c|c|c|c|c|c|c|c|c|c|c|c|c|c|}
\hline \multirow{2}{*}{$\begin{array}{l}\text { Entidad } \\
\text { federativa }\end{array}$} & \multicolumn{2}{|c|}{$\begin{array}{c}\text { Enfermedades } \\
\text { infecciosas intestinales } \\
\text { A00-A09 } \\
\end{array}$} & \multicolumn{2}{|c|}{$\begin{array}{c}\text { Infecciones } \\
\text { respiratorias agudas* } \\
\text { J00-J22 } 22^{\S} \\
\end{array}$} & \multicolumn{2}{|c|}{$\begin{array}{l}\text { Tuberculosis } \\
\text { pulmonar } \\
\text { A15-A16 } \\
\end{array}$} & \multicolumn{2}{|c|}{$\begin{array}{c}\text { SIDA } \\
\text { B20-B24 }\end{array}$} & \multicolumn{2}{|c|}{$\begin{array}{c}\text { Ciertas afecciones } \\
\text { perinatales } \\
\text { P00-P96,A33 } \\
\end{array}$} & \multicolumn{2}{|c|}{$\begin{array}{c}\text { Deficiencias } \\
\text { de la nutrición } \\
\text { E40-E64 } \\
\end{array}$} & \multicolumn{2}{|c|}{$\begin{array}{c}\text { Anemia } \\
\text { D50-D64 }\end{array}$} \\
\hline & Def & Tasa & Def & Tasa & $\overline{\text { Def }}$ & Tasa & Def & Tasa & Def & Tasa & Def & Tasa & $\overline{\text { Def }}$ & Tasa \\
\hline $\mathrm{N}$ acional & 2324 & 5.2 & 6646 & 15.7 & 897 & 2.3 & 714 & 1.4 & 7810 & 790.5 & 4632 & 11.5 & 622 & 1.6 \\
\hline Aguascalientes & 19 & 4.5 & 40 & 10.7 & 6 & 2.0 & 0 & 0.0 & 86 & 811.2 & 56 & 13.9 & 2 & 0.6 \\
\hline Baja California & 19 & 2.2 & 100 & 11.8 & 33 & 3.0 & 49 & 3.6 & 194 & 728.7 & 61 & 7.7 & 7 & 1.0 \\
\hline Baja California Sur & 5 & 2.9 & 25 & 17.9 & 1 & 0.9 & 2 & 0.9 & 34 & 771.9 & 7 & 4.9 & 1 & 0.4 \\
\hline Campeche & 12 & 5.3 & 28 & 11.5 & 4 & 1.5 & 4 & 1.1 & 43 & 571.7 & 24 & 11.4 & 5 & 2.2 \\
\hline Coahuila & 37 & 4.0 & 105 & 12.5 & 38 & 4.5 & 12 & 1.0 & 105 & 448.8 & 67 & 8.1 & 6 & 0.7 \\
\hline Colima & 7 & 2.7 & 33 & 14.9 & 4 & 1.7 & 6 & 2.1 & 25 & 490.8 & 34 & 15.0 & 1 & 0.4 \\
\hline Chiapas & 281 & 17.2 & 315 & 22.8 & 77 & 6.5 & 35 & 1.8 & 327 & 704.2 & 182 & 15.1 & 40 & 2.9 \\
\hline Chihuahua & 52 & 4.1 & 226 & 19.6 & 34 & 3.0 & 25 & 1.6 & 311 & 990.7 & 104 & 9.2 & 12 & 1.0 \\
\hline Distrito Federal & 138 & 3.3 & 792 & 18.4 & 30 & 0.7 & 69 & 1.4 & 660 & 959.4 & 271 & 6.1 & 26 & 0.6 \\
\hline Durango & 20 & 3.6 & 63 & 11.0 & 9 & 1.6 & 3 & 0.5 & 76 & 498.3 & 34 & 6.2 & 2 & 0.4 \\
\hline Guanajuato & 100 & 4.4 & 367 & 16.9 & 17 & 1.0 & 26 & 1.2 & 455 & 881.8 & 352 & 17.1 & 33 & 1.8 \\
\hline Guerrero & 96 & 6.7 & 99 & 7.6 & 36 & 3.1 & 26 & 1.9 & 118 & 332.7 & 149 & 11.5 & 23 & 1.8 \\
\hline Hidalgo & 43 & 3.9 & 164 & 15.9 & 20 & 2.2 & 9 & 0.8 & 174 & 775.7 & 85 & 8.5 & 15 & 1.6 \\
\hline Jalisco & 118 & 3.8 & 500 & 17.1 & 41 & 1.6 & 48 & 1.6 & 459 & 714.0 & 376 & 12.2 & 16 & 0.5 \\
\hline México & 308 & 5.5 & 1088 & 20.7 & 61 & 1.3 & 60 & 0.9 & 1399 & 1066.0 & 529 & 11.7 & 35 & 0.8 \\
\hline Michoacán & 85 & 4.4 & 237 & 12.3 & 20 & 1.3 & 11 & 0.6 & 228 & 556.4 & 213 & 11.5 & 31 & 1.7 \\
\hline Morelos & 31 & 4.2 & 76 & 10.9 & 10 & 1.6 & 17 & 2.2 & 118 & 765.0 & 109 & 16.3 & 8 & 1.3 \\
\hline $\mathrm{N}$ ayarit & 22 & 4.5 & 48 & 11.0 & 7 & 1.8 & 9 & 2.1 & 37 & 401.4 & 49 & 11.3 & 2 & 0.4 \\
\hline N uevo León & 34 & 2.2 & 155 & 10.3 & 36 & 2.3 & 18 & 0.8 & 203 & 541.3 & 69 & 4.7 & 4 & 0.3 \\
\hline 0 axaca & 217 & 13.1 & 287 & 17.6 & 70 & 5.0 & 35 & 2.1 & 300 & 806.2 & 362 & 22.5 & 69 & 4.5 \\
\hline Puebla & 182 & 7.2 & 543 & 21.5 & 55 & 2.7 & 44 & 1.8 & 704 & 1316.7 & 379 & 16.2 & 95 & 4.3 \\
\hline Q uerétaro & 28 & 4.6 & 90 & 15.4 & 13 & 3.1 & 6 & 0.9 & 147 & 969.5 & 97 & 18.2 & 7 & 1.6 \\
\hline Q uintana Roo & 9 & 3.0 & 15 & 7.2 & 2 & 0.9 & 8 & 1.7 & 75 & 732.1 & 11 & 7.2 & 2 & 1.7 \\
\hline San Luis Potosí & 72 & 6.8 & 138 & 13.3 & 25 & 2.8 & 6 & 0.6 & 159 & 690.0 & 129 & 12.6 & 18 & 1.9 \\
\hline Sinaloa & 40 & 3.8 & 110 & 10.7 & 30 & 2.9 & 13 & 1.0 & 81 & 319.7 & 87 & 8.7 & 10 & 1.1 \\
\hline Sonora & 27 & 3.1 & 136 & 15.9 & 18 & 2.1 & 7 & 0.6 & 170 & 746.2 & 95 & 11.6 & 17 & 2.1 \\
\hline Tabasco & 32 & 3.8 & 57 & 8.9 & 24 & 3.9 & 21 & 2.3 & 168 & 856.2 & 76 & 12.9 & 15 & 2.4 \\
\hline Tamaulipas & 27 & 2.0 & 77 & 6.9 & 31 & 2.5 & 16 & 1.0 & 139 & 492.3 & 71 & 6.6 & 8 & 0.7 \\
\hline Tlaxcala & 16 & 2.8 & 116 & 25.0 & 4 & 1.3 & 3 & 0.5 & 101 & 1007.8 & 65 & 14.9 & 9 & 2.3 \\
\hline Veracruz & 151 & 4.7 & 392 & 12.7 & 118 & 4.0 & 109 & 3.1 & 478 & 736.8 & 351 & 11.7 & 78 & 2.6 \\
\hline Yucatán & 59 & 7.7 & 96 & 12.5 & 16 & 2.4 & 13 & 1.5 & 107 & 667.4 & 81 & 10.5 & 17 & 2.3 \\
\hline Zacatecas & 37 & 5.5 & 128 & 19.2 & 7 & 1.3 & 4 & 0.6 & 129 & 902.2 & 57 & 8.0 & 8 & 1.2 \\
\hline
\end{tabular}

Los totales no incluyen defunciones de mexicanos que residían en el extranjero

Fuente: Instituto Nacional de Estadística, Geografía e Informática/Secretaría de Salud. Dirección General de Información en Salud. Base de datos de defunciones 2002. México, 2004 


\section{Cuadro VIII

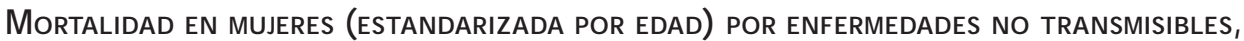 SEGÚN ENTIDAD fEDERATIVA DE RESIDENCIA HABITUAL. MéXICO, 2002}

\begin{tabular}{|c|c|c|c|c|c|c|c|c|c|c|c|c|c|c|c|c|c|c|c|c|}
\hline \multirow[b]{2}{*}{ Entidad federativa } & \multicolumn{2}{|c|}{$\begin{array}{l}\text { Enfermedad } \\
\text { isquémica } \\
\text { del corazón } \\
120-125^{\ddagger}\end{array}$} & \multicolumn{2}{|c|}{$\begin{array}{c}\text { Enfermedades } \\
\text { cerebro- } \\
\text { vasculares } \\
160-169^{\ddagger}\end{array}$} & \multicolumn{2}{|c|}{$\begin{array}{c}\text { Diabetes } \\
\text { Mellitus } \\
\text { E10-E14 }\end{array}$} & \multicolumn{2}{|c|}{$\begin{array}{l}\text { Cáncer } \\
\text { pulmonar } \\
\text { C33-C34 }\end{array}$} & \multicolumn{2}{|c|}{$\begin{array}{c}\text { Cáncer del } \\
\text { estómago } \\
\text { C16 }\end{array}$} & \multicolumn{2}{|c|}{$\begin{array}{c}\mathrm{CaCu}^{*} \\
\mathrm{C}^{3} 3^{\ddagger}\end{array}$} & \multicolumn{2}{|c|}{$\begin{array}{c}\text { Cáncer } \\
\text { de mama } \\
\text { C50 }\end{array}$} & \multicolumn{2}{|c|}{$\begin{array}{c}\text { Cirrosis y otras } \\
\text { enfermedades cró- } \\
\text { nicas del hígado } \\
\mathrm{K} 70, \mathrm{~K} 73, \mathrm{~K} 74, \mathrm{~K} 76^{\ddagger}\end{array}$} & \multicolumn{2}{|c|}{$\begin{array}{c}\text { Enfermedad } \\
\text { pulmonar } \\
\text { obstructiva } \\
J 44^{\ddagger}\end{array}$} & \multicolumn{2}{|c|}{$\begin{array}{l}\text { Malfor- } \\
\text { maciones } \\
\text { congénitas } \\
000-099 \ddagger\end{array}$} \\
\hline & Def & Tasa & Def & Tasa & Def & Tasa & Def & Tasa & Def & Tasa & $f$ & Tasa & Def & Tasa & Def & Tasa & Def & Tasa & Def & $T$ \\
\hline acional & 650 & 1.0 & 145 & 39.1 & 673 & 85.8 & 40 & 6.2 & 367 & 6.6 & 23 & 11.6 & 3837 & 10 & 985 & 16.8 & 5312 & 14.8 & 4198 & 7. \\
\hline . & 59 & 49.7 & 30 & 40.1 & 310 & VI. & 35 & 10.7 & 21 & 6.6 & 30 & 9.0 & 37 & 10. & 2 & 1.0 & 143 & 44.4 & 49 & 0.0 \\
\hline $\mathrm{jaC}$ & 97 & 89.4 & 292 & 43.1 & 601 & 88.5 & 81 & 12.1 & 28 & 4.0 & 114 & 13.4 & 104 & 11.9 & 92 & 12.3 & 85 & 13.0 & 104 & 7.0 \\
\hline $\mathrm{jaC}$ & 91 & 72.9 & 38 & 31.3 & 84 & 0.0 & 10 & 7.9 & 2 & 1.8 & 19 & 0.0 & 20 & 0.0 & 17 & 11.7 & 13 & 11.3 & 11 & $\overline{4.3}$ \\
\hline ampeche & 107 & 52.5 & 65 & 31.4 & 171 & 82.9 & 17 & 8.3 & 15 & 7.1 & 44 & 18.9 & 16 & 7.4 & 65 & 29.7 & 14 & 6.9 & 20 & 5.3 \\
\hline oahuila & 608 & 77.1 & 339 & 42.7 & 930 & 117.5 & 73 & 9.2 & 37 & 4.5 & 123 & 14.3 & 119 & 13.8 & 103 & 12.9 & 113 & 14.4 & 75 & 5.7 \\
\hline lima & 152 & 72.8 & 76 & 36.8 & 163 & 84.1 & 11 & 5.7 & J & 2.5 & 36 & 17.1 & 36 & 17. & 26 & 13.2 & 21 & 10.8 & 23 & 8.2 \\
\hline niapas & 493 & 48.9 & 377 & 36.6 & 783 & 77.3 & 46 & 4.5 & 136 & 13.1 & 199 & 17.3 & 60 & 5.3 & 257 & 23.6 & 92 & 9.3 & 130 & 5.0 \\
\hline nihuahua & 984 & 95.4 & 396 & 38.0 & 879 & 84.8 & 129 & 12.5 & 71 & 6.7 & 128 & 11.0 & 157 & 13.7 & 101 & 9.1 & 167 & 16.8 & 140 & 7.7 \\
\hline istrito $\mathrm{F}$ & 245 & 76.3 & 699 & 40.5 & 3733 & 94.3 & 229 & 5.8 & 275 & 6.7 & 345 & 8.4 & 574 & 14.1 & 519 & 13.3 & 583 & 13.9 & 378 & 9.5 \\
\hline uran & 17 & 60.7 & 166 & 31.2 & 407 & 78.9 & 30 & 5.7 & 30 & 5.7 & 58 & 10.6 & 46 & 8.3 & 52 & 10.0 & 63 & 11.8 & 29 & J.L \\
\hline danajuato & 955 & 53.9 & 759 & 42.7 & 670 & 103.5 & 98 & 6.1 & 98 & 6.0 & 179 & 10.7 & 177 & 10.4 & 193 & 11.6 & 383 & 21.6 & 292 & 9.9 \\
\hline uerrer & 400 & 35.5 & 331 & 29.3 & 604 & 55.8 & 46 & 4.1 & 98 & 9.0 & 148 & 13.5 & 62 & 5.4 & 107 & 9.9 & 88 & 7.9 & 68 & 3.7 \\
\hline idalgo & 477 & 53.1 & 346 & 38.7 & 581 & 69.5 & 42 & 5.0 & 59 & 6.8 & 60 & 7.1 & 61 & 6.9 & 224 & 26.6 & 93 & 10.3 & 56 & 4.3 \\
\hline isco & 555 & 62.6 & 34 & 39.6 & 000 & 83.3 & 20 & 9.1 & 140 & 5.5 & 231 & 9.3 & 332 & 13. & 339 & 14 & 592 & 22.3 & 280 & 7.8 \\
\hline éxico & 102 & 55.5 & 465 & 37.7 & 3903 & 100.0 & 163 & 4.1 & 232 & 5.8 & 462 & 10.2 & 476 & 10.3 & 093 & 25.3 & 812 & 21.6 & 675 & 9.0 \\
\hline ichoac & 807 & 47.9 & 624 & 37.2 & 1268 & 82.4 & 108 & 7.0 & 92 & 5.9 & 211 & 13.8 & 141 & 9.2 & 181 & 11.7 & 255 & 14.9 & 130 & 5.7 \\
\hline orelos & 319 & 51.1 & 190 & 30.8 & 502 & 84.0 & 30 & 5.1 & 47 & 7.6 & 104 & 16.4 & 57 & 8.9 & 119 & 19.6 & 97 & 15.8 & 52 & 5.8 \\
\hline ayarit & 226 & 59.5 & 160 & 40.6 & 251 & 70.3 & 17 & 4.5 & 15 & 4.0 & 53 & 14.1 & 33 & 0.9 & 31 & 8.5 & 52 & 13.0 & 30 & 5.8 \\
\hline 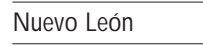 & 125 & 80.7 & 71 & 41.0 & 112 & 80.5 & 107 & 7.8 & 62 & 4.3 & 108 & 7.3 & 235 & 15. & 180 & 12.9 & 194 & 14.0 & 141 & 6.5 \\
\hline axaca & 2 & 37.7 & 532 & 36.7 & 848 & 62.4 & 37 & 2.7 & 126 & 9.0 & 221 & 16.2 & 68 & 5.0 & 281 & 20.6 & 146 & 10.0 & 156 & 7.4 \\
\hline iebla & 748 & 36.3 & 779 & 38.4 & 1708 & 90.5 & 53 & 2.8 & 128 & 6.6 & 227 & 11.6 & 148 & 7.6 & 535 & 27.9 & 203 & 10.0 & 330 & 10.7 \\
\hline uere & 192 & 42.8 & 90 & 42.0 & 383 & 88.8 & 18 & 4.0 & 24 & 5.5 & 42 & 9.0 & 40 & 8.3 & 92 & 19.6 & 113 & 24.4 & 85 & 10.0 \\
\hline uinta & 55 & 36.4 & 54 & 35.9 & 104 & 65.7 & 4 & 3.1 & 18 & 10.3 & 28 & 13.4 & 22 & 9.8 & 39 & 22.3 & 15 & 10.2 & 49 & 8.3 \\
\hline a $\mathrm{Lu}$ & 473 & 50.8 & 345 & 36.9 & 596 & 69.1 & 48 & 5.6 & 69 & 7.8 & 105 & 11.9 & 69 & 7.6 & 81 & 9.3 & 144 & 15.5 & 117 & 8.4 \\
\hline naloa & 706 & 77.8 & 309 & 34.2 & 650 & 74.3 & 91 & 10.5 & 61 & 6.7 & 96 & 10.0 & 100 & 10.4 & 66 & 7.3 & 80 & 8.8 & 56 & 3.8 \\
\hline nora & 715 & 94.6 & 284 & 36.9 & 576 & 76.6 & 73 & 9.6 & 59 & 7.7 & 87 & 10.2 & 105 & 12.8 & 80 & 10.1 & 103 & 13.7 & 68 & 5.2 \\
\hline basco & 300 & 57.8 & 229 & 43.7 & 537 & 102.4 & 32 & 5.5 & 49 & 8.7 & 87 & 14.0 & 47 & 7.5 & 78 & 14.4 & 48 & 9.6 & 83 & 7.6 \\
\hline nat & 702 & 70.3 & 375 & 36.9 & 885 & 88.1 & 84 & 8.4 & 55 & 5.2 & 140 & 12.6 & 150 & 13. & 106 & 10.3 & 113 & 11.3 & 30 & 5.0 \\
\hline laxcala & 115 & 30.8 & 117 & 31.7 & 329 & 97.6 & 10 & 3.0 & 15 & 4.2 & 36 & 10.0 & 30 & 8.8 & 76 & 22.3 & 35 & 9.0 & 57 & 0.1 \\
\hline eracruz & 1527 & 55.0 & 1274 & 46.4 & 2172 & 81.0 & 120 & 4.4 & 211 & 7.7 & 445 & 15.6 & 233 & 8.2 & 533 & 19.6 & 315 & 11.5 & 298 & 8.0 \\
\hline ucatán & 431 & 61.0 & 345 & 49.2 & 557 & 85.3 & 25 & 3.8 & 57 & 8.5 & 112 & 16.9 & 43 & 6.3 & 265 & 40.7 & 47 & 6.4 & 77 & 8.4 \\
\hline & 15 & & 94 & & 10 & & & 1.8 & 2 & 9.9 & & & $3 y$ & & 31 & & & & & \\
\hline
\end{tabular}

* CACU:Cáncer del cuello uterino

₹ Códigos de la CIE 10: Clasificación Estadística Internacional de Enfermedades y Problemas Relacionados con la Salud. 10a revisión

Tasa por 100000 habitantes estandarizada por el método directo usando la población mundial estándar (ver nota metodológica) Los totales no incluyen defunciones de mexicanos que residían en el extranjero

Fuente: Instituto Nacional de Estadística, Geografía e Informática/Secretaría de Salud. Dirección General de Información en Salud. Base de datos de defunciones 2002. México, 2004 


\section{Cuadro IX \\ Mortalidad EN MUJeres (estandaRIZADA POR EDAD) POR LESIONES, SEGÚN ENTIDAD FEDERATIVA De Residencia habitual. México, 2002}

\begin{tabular}{|c|c|c|c|c|c|c|c|c|c|c|c|c|}
\hline \multirow[b]{2}{*}{ Entidad federativa } & \multicolumn{2}{|c|}{$\begin{array}{c}\text { Total } \\
\text { lesiones } \\
\text { V01-Y98* }\end{array}$} & \multicolumn{2}{|c|}{$\begin{array}{c}\text { Accidentes de tráfico } \\
\text { vehículos de motor } \\
\neq\end{array}$} & \multicolumn{2}{|c|}{$\begin{array}{c}\text { Los } \\
\text { demás } \\
\text { accidentes }\end{array}$} & \multicolumn{2}{|c|}{$\begin{array}{l}\text { Homicidios } \\
\text { X85-Y09* }\end{array}$} & \multicolumn{2}{|c|}{$\begin{array}{c}\text { Suicidios } \\
\times 60-\times 84 *\end{array}$} & \multicolumn{2}{|c|}{$\begin{array}{c}\text { Las demás lesiones } \\
\text { intencionales } \\
\text { Y10-Y98* }\end{array}$} \\
\hline & Def & Tasa & Def & Tasa & Def & Tasa & Def & Tasa & Def & Tasa & Def & Tasa \\
\hline$N$ acional & 10803 & 23.5 & 3233 & 7.0 & 5140 & 11.6 & 1266 & 2.6 & 657 & 1.3 & 760 & 1.7 \\
\hline Aguascalientes & 131 & 29.8 & 38 & 8.1 & 80 & 18.8 & 6 & 1.4 & 7 & 1.5 & 7 & 2.0 \\
\hline Baja California & 339 & 28.2 & 59 & 5.1 & 206 & 17.2 & 33 & 2.6 & 17 & 1.3 & 27 & 2.5 \\
\hline Baja California Sur & 54 & 28.6 & 24 & 11.1 & 23 & 14.2 & 3 & 1.6 & 4 & 1.7 & 4 & 0.0 \\
\hline Campeche & 63 & 20.9 & 17 & 5.5 & 32 & 11.0 & 2 & 0.7 & 10 & 3.2 & 4 & 1.1 \\
\hline Coahuila & 239 & 23.3 & 71 & 6.4 & 133 & 13.8 & 17 & 1.4 & 18 & 1.6 & 5 & 0.6 \\
\hline Colima & 51 & 20.0 & 20 & 7.5 & 22 & 9.3 & 3 & 0.9 & 2 & 0.6 & 5 & 2.2 \\
\hline Chiapas & 384 & 23.2 & 29 & 1.4 & 253 & 16.2 & 79 & 4.5 & 11 & 0.6 & 18 & 1.1 \\
\hline Chihuahua & 496 & 33.8 & 113 & 7.9 & 278 & 19.1 & 76 & 4.9 & 28 & 2.0 & 4 & 0.3 \\
\hline Distrito Federal & 901 & 20.6 & 320 & 7.4 & 349 & 8.2 & 98 & 2.1 & 59 & 1.3 & 113 & 2.6 \\
\hline Durango & 165 & 23.9 & 42 & 5.6 & 90 & 13.8 & 20 & 2.5 & 12 & 1.7 & 2 & 0.3 \\
\hline Guanajuato & 530 & 23.2 & 185 & 8.2 & 253 & 11.3 & 40 & 1.7 & 42 & 1.5 & 25 & 1.2 \\
\hline Guerrero & 412 & 30.1 & 137 & 9.6 & 195 & 14.8 & 56 & 4.1 & 19 & 1.2 & 17 & 1.3 \\
\hline Hidalgo & 201 & 18.6 & 50 & 4.7 & 124 & 11.3 & 9 & 0.9 & 6 & 0.5 & 12 & 1.2 \\
\hline Jalisco & 825 & 26.4 & 298 & 9.7 & 366 & 11.7 & 65 & 2.1 & 56 & 1.7 & 64 & 2.0 \\
\hline México & 1358 & 23.6 & 444 & 7.8 & 502 & 9.4 & 314 & 4.8 & 69 & 1.0 & 61 & 1.3 \\
\hline Michoacán & 560 & 29.0 & 148 & 7.8 & 266 & 13.9 & 68 & 3.3 & 28 & 1.2 & 63 & 3.4 \\
\hline Morelos & 136 & 18.0 & 41 & 5.4 & 62 & 8.6 & 11 & 1.3 & 9 & 1.1 & 17 & 2.2 \\
\hline $\mathrm{N}$ ayarit & 111 & 24.9 & 39 & 9.0 & 47 & 10.2 & 16 & 3.7 & 5 & 1.0 & 5 & 1.2 \\
\hline Nuevo León & 326 & 18.3 & 83 & 4.5 & 201 & 11.7 & 17 & 0.9 & 21 & 1.0 & 8 & 0.5 \\
\hline 0 axaca & 403 & 24.6 & 112 & 6.9 & 195 & 11.8 & 62 & 3.9 & 15 & 0.8 & 34 & 2.1 \\
\hline Puebla & 589 & 24.2 & 182 & 7.4 & 283 & 11.7 & 62 & 2.7 & 32 & 1.2 & 37 & 1.5 \\
\hline Q uerétaro & 175 & 27.6 & 72 & 11.0 & 87 & 14.4 & 9 & 1.2 & 5 & 0.7 & 6 & 1.1 \\
\hline Q uintana Roo & 76 & 20.2 & 20 & 5.9 & 32 & 8.9 & 13 & 2.7 & 10 & 2.5 & 1 & 0.2 \\
\hline San Luis Potosí & 234 & 21.1 & 80 & 7.2 & 117 & 10.7 & 17 & 1.5 & 17 & 1.4 & 12 & 1.3 \\
\hline Sinaloa & 255 & 21.9 & 84 & 6.8 & 124 & 11.5 & 25 & 2.0 & 18 & 1.3 & 18 & 1.7 \\
\hline Sonora & 243 & 23.1 & 92 & 8.2 & 99 & 10.2 & 31 & 2.9 & 17 & 1.4 & 9 & 1.0 \\
\hline Tabasco & 185 & 20.8 & 58 & 5.8 & 76 & 9.7 & 14 & 1.6 & 25 & 2.5 & 15 & 1.8 \\
\hline Tamaulipas & 268 & 20.3 & 120 & 8.6 & 106 & 8.6 & 18 & 1.2 & 12 & 0.9 & 17 & 1.3 \\
\hline Tlaxcala & 132 & 28.8 & 35 & 7.7 & 73 & 16.4 & 12 & 2.2 & 8 & 1.7 & 10 & 2.4 \\
\hline Veracruz & 624 & 18.6 & 117 & 3.4 & 294 & 8.9 & 51 & 1.5 & 43 & 1.1 & 123 & 3.7 \\
\hline Yucatán & 146 & 18.6 & 44 & 5.4 & 69 & 9.1 & 4 & 0.6 & 22 & 2.6 & 13 & 1.6 \\
\hline Zacatecas & 191 & 28.4 & 59 & 9.5 & 103 & 14.8 & 15 & 2.3 & 10 & 1.2 & 4 & 0.5 \\
\hline
\end{tabular}

* Códigos de la CIE 10: Clasificación Estadística Internacional de Enfermedades y Problemas Relacionados con la Salud. 10a revisión

₹ Códigos de la CIE 10:V02-V04 (.1, .9),V09.2-V09.3,V09.9,V12-V14 (.3-.9),V19.4-V19.6,V20-V28 (.3-.9),V29-V79 (.4-.9), V80.3-V80.5,V81.1,V82.1,V83V86 (.0-.3), V87.0-V87.8,V89.2,V89.9, Y 85.0

Tasa por 100000 habitantes estandarizada por el método directo usando la población mundial estándar (ver nota metodológica) Los totales no incluyen defunciones de mexicanos que residían en el extranjero

Fuente: Instituto N acional de Estadística, Geografía e Informática/Secretaría de Salud. Dirección General de Información en Salud. Base de datos de defunciones 2002. México, 2004 
Cuadro X

MorTALIDAD EN HOMBRES (ESTANDARIZADA POR EDAD) POR ENFERMEDADES TRANSMISIBLES, PERINATALES Y DEFICIENCIAS DE LA NUTRICIÓN, SEGÚN ENTIDAD FEDERATIVA DE RESIDENCIA HABITUAL, 2002

\begin{tabular}{|c|c|c|c|c|c|c|c|c|c|c|c|c|c|c|}
\hline \multirow[b]{2}{*}{ Entidad federativa } & \multicolumn{2}{|c|}{$\begin{array}{c}\text { Entermedades } \\
\text { infecciosas intestinales } \\
\text { A00-A09\$ }\end{array}$} & \multicolumn{2}{|c|}{$\begin{array}{c}\text { Infecciones } \\
\text { respiratorias agudas* } \\
\text { J00-J22 } 22^{\S} \\
\end{array}$} & \multicolumn{2}{|c|}{$\begin{array}{l}\text { Tuberculosis } \\
\text { pulmonar } \\
\text { A15-A16 }\end{array}$} & \multicolumn{2}{|c|}{$\begin{array}{c}\text { SIDA } \\
\text { B20-B24 }\end{array}$} & \multicolumn{2}{|c|}{$\begin{array}{c}\text { Ciertas afecciones } \\
\text { perinatales } \\
\text { P00-p96,A33 }\end{array}$} & \multicolumn{2}{|c|}{$\begin{array}{c}\text { Deficiencias } \\
\text { de la nutrición } \\
\text { E40-E64 }\end{array}$} & \multicolumn{2}{|c|}{$\begin{array}{c}\text { Anemia } \\
\text { D50-D64 }\end{array}$} \\
\hline & Def & Tasa & Def & Tasa & Def & Tasa & Def & Tasa & Def & Tasa & Def & Tasa & Def & Tasa \\
\hline$N$ acional & 2349 & 5.6 & 7468 & 20.5 & 1790 & 5.4 & 3748 & 8.1 & 10716 & 1037.6 & 4280 & 13.1 & 588 & 1.9 \\
\hline Aguascalientes & 16 & 4.3 & 26 & 8.4 & 7 & 2.4 & 18 & 4.9 & 126 & 1138.0 & 38 & 13.6 & 6 & 2.5 \\
\hline Baja California & 37 & 3.4 & 166 & 19.7 & 128 & 13.1 & 205 & 15.2 & 286 & 1025.5 & 66 & 9.3 & 5 & 0.4 \\
\hline Baja California Sur & 3 & 2.0 & 20 & 16.9 & 10 & 7.1 & 20 & 8.5 & 50 & 1081.1 & 16 & 13.3 & 2 & 1.9 \\
\hline Campeche & 9 & 2.8 & 41 & 18.2 & 15 & 6.0 & 43 & 13.4 & 57 & 724.5 & 37 & 19.1 & 6 & 3.4 \\
\hline Coahuila & 41 & 4.6 & 122 & 17.1 & 65 & 9.2 & 66 & 5.9 & 176 & 718.6 & 72 & 11.0 & 6 & 1.0 \\
\hline Colima & 6 & 2.6 & 32 & 14.9 & 16 & 8.8 & 26 & 9.4 & 34 & 639.1 & 30 & 15.7 & 1 & 0.6 \\
\hline Chiapas & 301 & 21.6 & 357 & 28.3 & 140 & 12.9 & 118 & 6.4 & 459 & 946.8 & 171 & 15.8 & 39 & 4.2 \\
\hline Chihuahua & 47 & 3.8 & 277 & 27.2 & 57 & 5.4 & 137 & 8.8 & 415 & 1262.5 & 112 & 12.3 & 16 & 1.6 \\
\hline D istrito Federal & 130 & 3.7 & 741 & 21.2 & 55 & 1.6 & 480 & 10.8 & 817 & 1138.0 & 189 & 5.5 & 20 & 0.6 \\
\hline Durango & 15 & 2.8 & 59 & 11.7 & 35 & 7.8 & 34 & 5.5 & 150 & 941.5 & 40 & 8.3 & 5 & $\overline{1.1}$ \\
\hline Guanajuato & 107 & 4.9 & 394 & 22.7 & 30 & 2.2 & 96 & 5.1 & 683 & 1266.0 & 291 & 17.8 & 29 & 2.0 \\
\hline Guerrero & 101 & 7.8 & 123 & 11.1 & 68 & 6.8 & 148 & 11.4 & 193 & 520.7 & 136 & 12.6 & 16 & 1.6 \\
\hline Hidalgo & 36 & 3.2 & 172 & 21.2 & 27 & 3.7 & 36 & 3.6 & 266 & 1135.1 & 84 & 11.3 & 12 & 1.6 \\
\hline Jalisco & 85 & 3.0 & 532 & 21.8 & 80 & 3.7 & 299 & 10.1 & 605 & 900.0 & 312 & 13.3 & 18 & 0.8 \\
\hline México & 333 & 6.4 & 1345 & 27.1 & 96 & 2.7 & 350 & 5.4 & 1780 & 1297.4 & 467 & 11.8 & 33 & 0.9 \\
\hline Michoacán & 81 & 4.7 & 245 & 15.4 & 36 & 2.5 & 92 & 5.7 & 361 & 842.9 & 202 & 13.4 & 31 & 2.1 \\
\hline Morelos & 31 & 4.2 & 77 & 12.9 & 23 & 4.5 & 79 & 10.9 & 157 & 974.1 & 91 & 16.7 & 10 & 1.9 \\
\hline$N$ ayarit & 21 & 5.2 & 57 & 16.4 & 32 & 9.3 & 42 & 9.4 & 43 & 446.0 & 46 & 12.3 & 8 & 2.5 \\
\hline Nuevo León & 33 & 2.2 & 236 & 19.5 & 110 & 7.9 & 133 & 6.8 & 275 & 701.0 & 70 & 6.2 & 7 & 0.7 \\
\hline 0 axaca & 242 & 16.0 & 321 & 23.3 & 87 & 7.2 & 102 & 7.0 & 429 & 1103.8 & 293 & 23.1 & 70 & 5.5 \\
\hline Puebla & 201 & 7.7 & 613 & 27.6 & 83 & 5.0 & 182 & 8.0 & 1043 & 1867.1 & 343 & 18.6 & 58 & 3.4 \\
\hline Q uerétaro & 32 & 6.1 & 86 & 17.5 & 18 & 4.3 & 21 & 3.6 & 165 & 1041.7 & 98 & 23.2 & 7 & 1.3 \\
\hline Q uintana Roo & 9 & 1.6 & 28 & 12.6 & 6 & 4.2 & 55 & 11.4 & 111 & 1035.6 & 15 & 11.7 & 2 & 1.7 \\
\hline San Luis Potosí & 54 & 5.3 & 160 & 18.6 & 58 & 7.8 & 42 & 4.4 & 229 & 950.7 & 133 & 17.1 & 16 & 1.9 \\
\hline Sinaloa & 25 & 2.6 & 120 & 13.0 & 59 & 6.6 & 73 & 5.6 & 111 & 418.8 & 94 & 10.7 & 5 & 0.6 \\
\hline Sonora & 27 & 2.6 & 157 & 20.5 & 63 & 7.6 & 62 & 5.3 & 225 & 943.6 & 101 & 14.4 & 14 & 1.9 \\
\hline Tabasco & 32 & 3.4 & 89 & 14.3 & 54 & 9.8 & 113 & 12.9 & 199 & 970.4 & 69 & 13.0 & 13 & 2.7 \\
\hline Tamaulipas & 27 & 2.2 & 100 & 10.9 & 68 & 6.9 & 90 & 5.9 & 148 & 501.2 & 87 & 9.9 & 8 & 0.8 \\
\hline Tlaxcala & 31 & 7.0 & 112 & 27.6 & 5 & 1.7 & 26 & 5.1 & 163 & 1557.9 & 56 & 18.3 & 7 & 2.0 \\
\hline Veracruz & 149 & 4.7 & 430 & 16.2 & 211 & 8.5 & 471 & 14.4 & 676 & 997.1 & 358 & 14.4 & 97 & 4.1 \\
\hline Yucatán & 63 & 9.5 & 116 & 19.1 & 39 & 6.5 & 69 & 8.5 & 126 & 751.7 & 106 & 18.0 & 11 & 2.0 \\
\hline Zacatecas & 24 & 3.8 & 114 & 20.4 & 9 & 1.8 & 20 & 3.8 & 158 & 1058.6 & 57 & 10.5 & 10 & 1.9 \\
\hline $\begin{array}{l}\text { Incluye ne } \\
\text { Tasa por } 1 \\
\text { Tasa por } 1 \\
\text { Códigos d }\end{array}$ & ilue & - & ano & Lal & da) & & 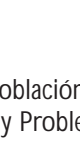 & 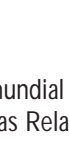 & (2) & & & & & \\
\hline
\end{tabular}

Los totales no incluyen defunciones de mexicanos que residían en el extranjero

Fuente: Instituto Nacional de Estadística, Geografía e Informática/Secretaría de Salud. Dirección General de Información en Salud. Base de datos de defunciones 2002. México, 2004 


\section{Cuadro XI}

\section{MoRTALIDAD EN HOMBRes (eSTANDARIZADA POR EDAD) POR ENFERMEDADES CRóNICAS, SEgún entidad federativa de residencia habitual del fallecido. México, 2002}

\begin{tabular}{|c|c|c|c|c|c|c|c|c|c|}
\hline & $\begin{array}{l}\text { Enfermedad } \\
\text { isquémica } \\
\text { del corazón } \\
120-125^{*}\end{array}$ & $\begin{array}{c}\text { Enfermedades } \\
\text { cerebro- } \\
\text { vasculares } \\
160-169 * \\
\end{array}$ & $\begin{array}{c}\text { Diabetes } \\
\text { mellitus } \\
\text { E10-E14* }\end{array}$ & $\begin{array}{l}\text { Cáncer } \\
\text { pulmonar } \\
\text { C33-C34* }\end{array}$ & $\begin{array}{c}\text { Cáncer del } \\
\text { estómago } \\
\text { C16* }\end{array}$ & $\begin{array}{l}\text { Cáncer de } \\
\text { próstata } \\
\text { C61* }\end{array}$ & $\begin{array}{c}\text { Cirrosis y otras } \\
\text { enfermedades } \\
\text { crónicas del hígado } \\
\mathrm{K} 70, \mathrm{~K} 73, \mathrm{~K} 74, \mathrm{~K} 76^{*}\end{array}$ & $\begin{array}{c}\text { Enfermedad } \\
\text { pulmonar } \\
\text { obstructiva } \\
\text { J44* }\end{array}$ & $\begin{array}{c}\text { Malformaciones } \\
\text { congénitas } \\
\text { Q00-Q99* }\end{array}$ \\
\hline tidad federativa & Def Tasa & Def Tasa & Def Tasa & Def Tasa & $\overline{\text { Def Tasa }}$ & $\overline{\text { Def Tasa }}$ & Def Tasa & Def Tasa & Def Tasa \\
\hline ior & $26618 \quad 90.0$ & $12371 \quad 42.1$ & 514184.2 & $4536 \quad 15.7$ & $2747 \quad 9.1$ & $4218 \quad 15.1$ & $9661 \quad 58.3$ & $6682 \quad 23.6$ & 4901 \\
\hline
\end{tabular}

\begin{tabular}{|c|c|c|c|c|c|c|c|c|c|c|c|c|c|c|}
\hline guascalientes & 218 & 87.5 & 115 & 45.7 & 241 & 97.4 & $43 \quad 18.6$ & $12 \quad 4.7$ & 4619.7 & 114 & 38.7 & 146 & 60.9 & 6310.1 \\
\hline Baja California & 911 & 140.3 & 319 & 48.2 & 561 & 84.6 & 12520.3 & 6910.8 & $89 \quad 15.2$ & 374 & 45.7 & 146 & 24.3 & $119 \quad 7.4$ \\
\hline Baja C alifornia Sur & 170 & 140.9 & 44 & 37.8 & 91 & 0.0 & 5250.3 & $\begin{array}{ll}6 & 6.0\end{array}$ & $24 \quad 0.0$ & 36 & 26.3 & 26 & 25.8 & $\begin{array}{ll}23 & 8.7\end{array}$ \\
\hline Campeche & 153 & 80.9 & 70 & 38.5 & 139 & 71.2 & 2915.2 & 2915.1 & $40 \quad 22.2$ & 112 & 49.6 & 27 & 15.5 & $34 \quad 7.8$ \\
\hline Coahuila & 876 & 127.9 & 346 & 50.6 & 719 & 100.5 & 19228.7 & $\begin{array}{ll}64 & 9.2 \\
\end{array}$ & 10015.5 & 322 & 41.2 & 185 & 28.5 & $85 \quad 6.2$ \\
\hline Colima & 207 & 117.1 & 60 & 34.4 & 135 & 77.7 & 3520.1 & 2011.5 & $38 \quad 22.5$ & 94 & 46.0 & 41 & 24.3 & 3010.0 \\
\hline Chiapas & 629 & 72.0 & 380 & 43.5 & 598 & 66.2 & $\begin{array}{ll}86 & 9.6 \\
\end{array}$ & 15216.3 & 12115.6 & 691 & 61.9 & 142 & 17.5 & $150 \quad 5.5$ \\
\hline Chihuahua & 1318 & 146.8 & 422 & 47.8 & 675 & 73.2 & 25829.6 & 10211.0 & 14117.3 & 405 & 35.6 & 288 & 34.8 & $154 \quad 8.2$ \\
\hline Distrito Federal & 3016 & 95.0 & 1162 & 36.6 & 3345 & 106.1 & $344 \quad 11.1$ & $254 \quad 8.0$ & 46515.2 & 1594 & 45.0 & 555 & 17.9 & $444 \quad 10.8$ \\
\hline Durango & 453 & 100.6 & 166 & 36.9 & 345 & 75.2 & $89 \quad 19.4$ & 5111.1 & $58 \quad 13.7$ & 151 & 29.4 & 145 & 33.4 & $58 \quad 6.4$ \\
\hline Guanajuato & 1120 & 83.5 & 582 & 42.9 & 1383 & 105.9 & 17413.6 & $104 \quad 7.7$ & 21316.3 & 829 & 56.8 & 460 & 34.2 & $\begin{array}{ll}298 & 9.7 \\
\end{array}$ \\
\hline Guerrero & 493 & 53.5 & 301 & 32.9 & 505 & 54.7 & $75 \quad 8.0$ & 9810.3 & $97 \quad 10.9$ & 400 & 40.7 & 135 & 15.3 & $\begin{array}{ll}65 & 3.2 \\
\end{array}$ \\
\hline Hidalgo & 532 & 77.7 & 322 & 47.2 & 472 & 68.7 & $\begin{array}{ll}43 & 6.4 \\
\end{array}$ & $55 \quad 8.0$ & 8012.1 & 709 & 95.0 & 86 & 12.8 & $\begin{array}{ll}65 & 4.8 \\
\end{array}$ \\
\hline alisco & 1912 & 90.2 & 867 & 40.7 & 821 & 88.5 & 39219.2 & $172 \quad 8.1$ & 39518.9 & 1081 & 47.4 & 655 & 30.8 & $\begin{array}{ll}350 & 9.4 \\
\end{array}$ \\
\hline éxico & 2414 & 73.6 & 1184 & 36.7 & 3439 & 97.0 & $293 \quad 9.0$ & $240 \quad 6.9$ & $367 \quad 12.5$ & 3340 & 79.6 & 814 & 27.7 & $774 \quad 10.0$ \\
\hline Michoacán & 917 & 68.2 & 505 & 36.9 & 1040 & 80.7 & 20015.5 & $111 \quad 8.5$ & 24218.3 & 594 & 44.1 & 307 & 22.4 & $154 \quad 6.7$ \\
\hline Morelos & 407 & 77.9 & 176 & 33.8 & 394 & 74.6 & $60 \quad 12.0$ & $\begin{array}{ll}52 & 9.3 \\
\end{array}$ & $72 \quad 14.4$ & 334 & 57.9 & 126 & 25.1 & $\begin{array}{ll}81 & 9.1 \\
\end{array}$ \\
\hline ayarit & 300 & 91.3 & 117 & 35.5 & 209 & 64.6 & 8226.1 & 3510.7 & $68 \quad 21.6$ & 116 & 33.5 & 100 & 31.1 & $35 \quad 6.6$ \\
\hline Nuevo León & 1607 & 136.4 & 575 & 50.1 & 866 & 72.4 & $322 \quad 27.8$ & $96 \quad 8.0$ & 12711.8 & 476 & 35.7 & 321 & 30.4 & $\begin{array}{ll}178 & 8.1 \\
\end{array}$ \\
\hline 0 axaca & 606 & 52.6 & 385 & 33.2 & 683 & 60.2 & $80 \quad 7.0$ & 13211.3 & $113 \quad 9.9$ & 1058 & 89.7 & 126 & 10.7 & $167 \quad 7.8$ \\
\hline Puebla & 925 & 59.4 & 633 & 40.6 & 1487 & 96.1 & $106 \quad 6.9$ & 1207.8 & 16811.3 & 1831 & 109.0 & 209 & 13.9 & $385 \quad 12.0$ \\
\hline Q uerétaro & 281 & 76.1 & 142 & 39.8 & 323 & 85.4 & $\begin{array}{ll}36 & 9.8 \\
\end{array}$ & $27 \quad 6.9$ & $56 \quad 16.5$ & 420 & 96.5 & 130 & 36.6 & 9710.8 \\
\hline Q uintana Roo & 158 & 94.5 & 50 & 37.4 & 96 & 56.8 & 2315.7 & 1810.0 & $10 \quad 9.2$ & 125 & 56.3 & 26 & 21.3 & $57 \quad 9.2$ \\
\hline San Luis Potosí & 546 & 76.1 & 319 & 44.4 & 484 & 67.4 & 11115.7 & $61 \quad 8.5$ & 7911.3 & 347 & 45.3 & 204 & 28.8 & $125 \quad 8.8$ \\
\hline Sinaloa & 967 & 117.9 & 327 & 39.5 & 572 & 70.0 & $302 \quad 37.4$ & $\begin{array}{ll}76 & 9.1 \\
\end{array}$ & 14318.4 & 181 & 20.5 & 204 & 25.9 & $\begin{array}{ll}79 & 5.1 \\
\end{array}$ \\
\hline Sonora & 1000 & 143.9 & 313 & 45.2 & 595 & 84.7 & 24735.6 & 9313.3 & 12219.1 & 264 & 32.9 & 223 & 34.9 & $94 \quad 6.9$ \\
\hline Tabasco & 398 & 85.8 & 282 & 61.5 & 406 & 85.3 & $85 \quad 18.4$ & 5111.0 & $74 \quad 18.1$ & 267 & 46.4 & 81 & 18.9 & $108 \quad 9.4$ \\
\hline Tamaulipas & 977 & 110.6 & 368 & 41.9 & 822 & 90.9 & 21724.7 & $\begin{array}{ll}75 & 8.4 \\
\end{array}$ & 12114.6 & 350 & 35.3 & 214 & 25.6 & $79 \quad 4.9$ \\
\hline Tlaxcala & 149 & 50.2 & 137 & 47.6 & 263 & 94.1 & $16 \quad 5.6$ & $22 \quad 7.4$ & 4014.6 & 223 & 73.2 & 56 & 20.0 & $\begin{array}{ll}51 & 8.9 \\
\end{array}$ \\
\hline Veracruz & 1903 & 84.3 & 1190 & 53.3 & 1864 & 80.6 & $276 \quad 12.1$ & $234 \quad 9.9$ & 35116.4 & 2226 & 86.6 & 304 & 14.1 & $\begin{array}{ll}342 & 8.7 \\
\end{array}$ \\
\hline Yucatán & 619 & 112.4 & 320 & 58.8 & 306 & 55.8 & $\begin{array}{ll}62 & 11.6 \\
\end{array}$ & 6011.1 & $\begin{array}{ll}69 & 12.9 \\
\end{array}$ & 448 & 74.6 & 85 & 15.8 & 10010.4 \\
\hline catece & 436 & 93.3 & 192 & 41.0 & 262 & 58.8 & $\begin{array}{ll}81 & 17.8\end{array}$ & $\begin{array}{ll}56 & 12.3\end{array}$ & $\begin{array}{ll}89 & 19.2\end{array}$ & 149 & 32.8 & 115 & 24.0 & 57 \\
\hline
\end{tabular}

* Códigos de la CIE 10: C lasificación Estadística Internacional de Enfermedades y Problemas Relacionados con la Salud. 10a revisión

Tasa por 100000 habitantes estandarizada por el método directo usando la población mundial estándar (ver nota metodológica) Los totales no incluyen defunciones de mexicanos que residían en el extranjero

Fuente: Instituto Nacional de Estadística, Geografía e Informática/Secretaría de Salud. Dirección General de Información en Salud. Base de datos de defunciones 2002. México, 2004 


\section{Cuadro XII}

MORTALIDAD EN HOMBRES (ESTANDARIZADA POR EDAD) POR LESIONES, SEgún entidad federativa de Residencia habitual del fallecido. México, 2002

\begin{tabular}{|c|c|c|c|c|c|c|c|c|c|c|c|c|}
\hline \multirow[b]{2}{*}{ Entidad federativa } & \multicolumn{2}{|c|}{$\begin{array}{c}\text { Total } \\
\text { Lesiones } \\
\text { V01-Y98* }\end{array}$} & \multicolumn{2}{|c|}{$\begin{array}{c}\text { Accidentes de tráfico } \\
\text { vehículos de motor } \\
\vdots\end{array}$} & \multicolumn{2}{|c|}{$\begin{array}{c}\text { Los } \\
\text { demás } \\
\text { accidentes }\end{array}$} & \multicolumn{2}{|c|}{$\begin{array}{l}\text { Homicidios } \\
\text { X85-Y09* }\end{array}$} & \multicolumn{2}{|c|}{$\begin{array}{c}\text { Suicidios } \\
\times 60-\times 84 *\end{array}$} & \multicolumn{2}{|c|}{$\begin{array}{l}\text { Las demás lesiones } \\
\text { intencionales } \\
\text { Y10-Y98* }\end{array}$} \\
\hline & Def & Tasa & Def & Tasa & Def & Tasa & Def & Tasa & Def & $\overline{\text { Tasa }}$ & Def & Tasa \\
\hline$N$ acional & 40854 & 92.2 & 11126 & 24.7 & 15521 & 36.6 & 8697 & 18.6 & 3189 & 6.8 & 2603 & 6.3 \\
\hline A guascalientes & 373 & 100.4 & 135 & 35.5 & 178 & 49.5 & 21 & 5.5 & 37 & 9.6 & 7 & 2.4 \\
\hline Baja California & 1392 & 113.0 & 230 & 19.2 & 637 & 53.8 & 369 & 27.5 & 88 & 7.1 & 69 & 5.5 \\
\hline Baja California Sur & 195 & 88.1 & 77 & 34.9 & 47 & 23.5 & 32 & 12.5 & 35 & 15.1 & 7 & 0.0 \\
\hline Campeche & 317 & 104.3 & 73 & 24.4 & 121 & 42.1 & 49 & 16.4 & 60 & 17.0 & 18 & 6.9 \\
\hline Coahuila & 851 & 76.5 & 243 & 21.7 & 370 & 35.6 & 121 & 9.7 & 108 & 8.8 & 14 & 1.5 \\
\hline Colima & 230 & 94.9 & 69 & 27.7 & 82 & 35.6 & 42 & 16.3 & 31 & 12.6 & 9 & 4.6 \\
\hline Chiapas & 1762 & 110.1 & 104 & 6.3 & 1164 & 74.4 & 395 & 23.4 & 54 & 3.3 & 57 & 4.3 \\
\hline Chihuahua & 1971 & 132.5 & 335 & 22.6 & 887 & 61.8 & 549 & 34.6 & 186 & 12.6 & 14 & 0.9 \\
\hline Distrito Federal & 2981 & 71.6 & 874 & 21.1 & 702 & 18.3 & 725 & 15.9 & 260 & 6.0 & 450 & 11.2 \\
\hline Durango & 663 & 101.0 & 141 & 20.8 & 280 & 45.0 & 166 & 24.4 & 72 & 10.0 & 6 & 1.2 \\
\hline Guanajuato & 1799 & 90.2 & 745 & 36.5 & 673 & 35.4 & 167 & 8.1 & 161 & 7.5 & 73 & 3.9 \\
\hline Guerrero & 1480 & 118.5 & 308 & 22.9 & 541 & 45.3 & 555 & 44.4 & 56 & 4.3 & 32 & 2.9 \\
\hline Hidalgo & 825 & 85.8 & 210 & 21.0 & 451 & 48.4 & 64 & 6.6 & 42 & 4.1 & 58 & 5.8 \\
\hline Jalisco & 2712 & 91.9 & 1013 & 34.3 & 874 & 30.3 & 417 & 13.8 & 265 & 8.5 & 171 & 6.1 \\
\hline México & 4899 & 82.0 & 1413 & 23.4 & 1403 & 25.5 & 1731 & 27.4 & 241 & 3.6 & 146 & 2.7 \\
\hline Michoacán & 2011 & 115.7 & 522 & 28.6 & 702 & 40.9 & 475 & 28.2 & 126 & 6.9 & 198 & 11.9 \\
\hline Morelos & 601 & 83.8 & 158 & 22.0 & 194 & 28.7 & 160 & 20.8 & 22 & 2.9 & 71 & 10.2 \\
\hline N ayarit & 546 & 123.0 & 206 & 45.8 & 167 & 38.4 & 125 & 28.4 & 32 & 6.8 & 17 & 3.9 \\
\hline N uevo León & 1140 & 62.2 & 235 & 12.5 & 692 & 38.7 & 83 & 4.1 & 117 & 6.2 & 22 & 1.5 \\
\hline 0 axaca & 1684 & 116.1 & 343 & 23.0 & 644 & 45.5 & 538 & 37.0 & 76 & 4.9 & 92 & 6.5 \\
\hline Puebla & 1898 & 86.9 & 602 & 27.4 & 788 & 37.0 & 311 & 13.9 & 106 & 4.2 & 104 & 5.2 \\
\hline Q uerétaro & 562 & 91.7 & 234 & 37.2 & 214 & 37.0 & 58 & 8.5 & 34 & 4.9 & 31 & 6.0 \\
\hline Q uintana Roo & 400 & 109.6 & 96 & 25.7 & 162 & 49.0 & 54 & 13.2 & 40 & 8.5 & 50 & 15.1 \\
\hline San Luis Potosí & 935 & 94.4 & 324 & 32.4 & 346 & 36.9 & 139 & 13.0 & 105 & 10.0 & 32 & 3.6 \\
\hline Sinaloa & 1279 & 105.2 & 364 & 30.0 & 365 & 32.3 & 442 & 33.9 & 75 & 6.2 & 35 & 3.1 \\
\hline Sonora & 1021 & 93.8 & 324 & 29.6 & 373 & 36.0 & 177 & 14.7 & 143 & 13.0 & 10 & 1.1 \\
\hline Tabasco & 964 & 112.4 & 276 & 32.4 & 341 & 40.4 & 86 & 9.6 & 153 & 18.0 & 115 & 13.3 \\
\hline Tamaulipas & 1037 & 76.1 & 374 & 26.5 & 332 & 25.9 & 163 & 11.1 & 99 & 7.5 & 72 & 5.5 \\
\hline Tlaxcala & 422 & 104.2 & 134 & 31.2 & 196 & 50.2 & 41 & 9.7 & 21 & 4.4 & 38 & 11.4 \\
\hline Veracruz & 2572 & 83.0 & 469 & 14.9 & 1081 & 35.1 & 316 & 10.0 & 183 & 5.8 & 544 & 18.2 \\
\hline Yucatán & 668 & 90.3 & 222 & 28.4 & 265 & 37.8 & 42 & 6.0 & 108 & 13.6 & 35 & 5.2 \\
\hline Zacatecas & 664 & 112.5 & 273 & 46.3 & 249 & 43.1 & 84 & 13.4 & 53 & 8.7 & 6 & 1.2 \\
\hline
\end{tabular}

* Códigos de la CIE 10: Clasificación Estadística Internacional de Enfermedades y Problemas Relacionados con la Salud. 10a revisión

₹ Códigos CIE-10:V02-V04 (.1, .9),V09.2-V09.3,V09.9,V12-V14 (.3-.9),V19.4-V19.6,V20-V28 (.3-.9),V29-V79 (.4-.9), V80.3-V80.5,V81.1,V82.1,V83-V86 (.0.3), V87.0-V87.8,V89.2,V89.9, Y 85.0

Tasa por 100000 habitantes estandarizada por el método directo usando la población mundial estándar (ver nota metodológica) Los totales no incluyen defunciones de mexicanos que residían en el extranjero

Fuente: Instituto Nacional de Estadística, Geografía e Informática/Secretaría de Salud. Dirección General de Información en Salud. Base de datos de defunciones 2002. México, 2004 


\section{Cuadro XIII}

INDICADORES DE CALIDAD DEL REGISTRO DE DEFUNCIONES, DE UNO U OTRO SEXO. MÉXICO, 2002

\begin{tabular}{|c|c|c|c|c|c|c|}
\hline Entidad federativa & $\begin{array}{c}\text { Porcentaje } \\
\text { de defunciones } \\
\text { de registro } \\
\text { extemporáneo* }\end{array}$ & $\begin{array}{l}\text { Porcentaje } \\
\text { de defunciones } \\
\text { certificadas } \\
\text { por médico }\end{array}$ & $\begin{array}{c}\text { Porcentaje } \\
\text { de defunciones } \\
\text { certificadas } \\
\text { por médico tratante }{ }^{\ddagger}\end{array}$ & $\begin{array}{c}\text { Porcentaje } \\
\text { de defunciones } \\
\text { ocurridas } \\
\text { en unidad médica }\end{array}$ & $\begin{array}{c}\text { Porcentaje } \\
\text { de defunciones en las } \\
\text { que no se especifica } \\
\text { la edad\$ }\end{array}$ & $\begin{array}{c}\text { Porcentaje } \\
\text { de defunciones en las } \\
\text { que no se especifica } \\
\text { la derechohabiencia }\end{array}$ \\
\hline N acional & 2.1 & 98.0 & 28.2 & 45.2 & 0.6 & 7.8 \\
\hline Aguascalientes & 1.0 & 99.4 & 35.5 & 56.8 & 0.7 & 3.2 \\
\hline Baja California & 1.7 & 99.7 & 25.3 & 59.5 & 0.9 & 12.0 \\
\hline Baja C alifornia Sur & 0.8 & 99.1 & 23.9 & 58.3 & 1.1 & 4.1 \\
\hline Campeche & 1.3 & 97.0 & 28.9 & 47.0 & 1.1 & 6.5 \\
\hline Coahuila & 3.7 & 99.0 & 26.7 & 57.9 & 0.8 & 8.6 \\
\hline Colima & 0.8 & 99.2 & 27.3 & 55.7 & 5.4 & 4.6 \\
\hline Chiapas & 0.7 & 88.3 & 15.0 & 26.0 & 1.1 & 19.9 \\
\hline Chihuahua & 6.2 & 96.1 & 19.3 & 53.7 & 1.4 & 25.7 \\
\hline Distrito Federal & 0.6 & 99.9 & 23.3 & 54.4 & 0.0 & 3.4 \\
\hline Durango & 9.0 & 96.7 & 26.1 & 48.8 & 0.4 & 15.5 \\
\hline Guanajuato & 2.1 & 99.4 & 37.6 & 40.2 & 0.7 & 6.5 \\
\hline Guerrero & 8.6 & 98.8 & 20.8 & 34.0 & 0.6 & 13.1 \\
\hline Hidalgo & 3.2 & 99.4 & 32.0 & 39.1 & 0.6 & 6.8 \\
\hline Jalisco & 0.6 & 99.6 & 34.0 & 50.9 & 0.5 & 6.5 \\
\hline México & 0.8 & 99.8 & 26.4 & 47.0 & 0.1 & 3.7 \\
\hline Michoacán & 3.0 & 99.0 & 42.9 & 35.9 & 0.4 & 7.8 \\
\hline Morelos & 2.3 & 99.4 & 32.9 & 45.5 & 1.0 & 4.0 \\
\hline $\mathrm{N}$ ayarit & 5.4 & 98.2 & 25.5 & 46.5 & 0.6 & 8.8 \\
\hline Nuevo León & 2.6 & 99.6 & 30.3 & 60.9 & 1.1 & 7.3 \\
\hline 0 axaca & 1.0 & 90.5 & 25.4 & 22.9 & 0.6 & 9.5 \\
\hline Puebla & 0.8 & 93.6 & 35.2 & 33.6 & 0.2 & 7.6 \\
\hline Q uerétaro & 1.4 & 98.5 & 29.7 & 45.8 & 0.7 & 3.9 \\
\hline Q uintana Roo & 2.1 & 98.2 & 21.1 & 54.4 & 3.5 & 14.7 \\
\hline San Luis Potosí & 3.0 & 98.7 & 31.2 & 40.7 & 0.2 & 6.7 \\
\hline Sinaloa & 4.9 & 98.9 & 21.7 & 54.2 & 0.4 & 9.2 \\
\hline Sonora & 0.9 & 99.4 & 19.9 & 58.4 & 0.4 & 8.1 \\
\hline Tabasco & 0.9 & 99.3 & 25.0 & 41.0 & 0.5 & 5.9 \\
\hline Tamaulipas & 5.0 & 99.3 & 26.1 & 56.1 & 0.8 & 6.6 \\
\hline Tlaxcala & 0.9 & 99.5 & 39.3 & 36.5 & 0.4 & 5.7 \\
\hline Veracruz & 2.3 & 98.3 & 29.2 & 36.6 & 0.6 & 6.1 \\
\hline Yucatán & 0.6 & 99.4 & 21.1 & 46.7 & 0.5 & 4.6 \\
\hline Zacatecas & 2.8 & 97.0 & 36.1 & 38.7 & 0.5 & 10.7 \\
\hline
\end{tabular}

* Se refiere a las defunciones ocurridas en años anteriores o no especificado al de registro

₹ $\mathrm{N}$ o se incluyen las muertes por causas violentas

$\S$ Incluye los no especificados en años y días

Fuente: Instituto N acional de Estadística, Geografía e Informática/Secretaría de Salud. Dirección General de Información en Salud. Base de datos de defunciones 2002. México, 2004 


\section{Cuadro XIV}

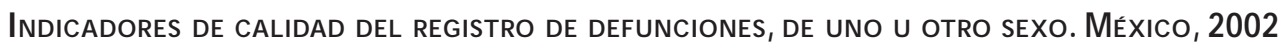

\begin{tabular}{|c|c|c|c|c|c|}
\hline Entidad federativa & $\begin{array}{l}\text { Mal definidas* } \\
\text { R00-R9g } \neq\end{array}$ & $\begin{array}{l}\text { Tumores de sitio mal definido } \neq \\
\text { C78-C80,C97 } \neq\end{array}$ & $\begin{array}{c}\text { Mal definidas de causa externa } \\
\text { Y10-Y3 } 34^{\ddagger}\end{array}$ & $\begin{array}{l}\text { Insuficiencia cardiaca\# } \\
150^{\neq}\end{array}$ & $\begin{array}{l}\text { Insuficiencia hepática\& } \\
\qquad 72^{\neq}\end{array}$ \\
\hline N acional & 2.0 & 4.9 & 5.4 & 11.27 & 8.28 \\
\hline Aguascalientes & 1.0 & 4.5 & 0.4 & 10.54 & 12.58 \\
\hline Baja California & 1.7 & 9.4 & 5.3 & 6.14 & 7.95 \\
\hline Baja C alifornia Sur & 0.8 & 4.4 & 1.6 & 8.14 & 6.78 \\
\hline Campeche & 3.0 & 5.2 & 4.2 & 11.73 & 9.14 \\
\hline Coahuila & 2.2 & 4.8 & 0.8 & 8.88 & 3.75 \\
\hline Colima & 1.4 & 4.4 & 2.8 & 7.08 & 6.02 \\
\hline Chiapas & 7.5 & 5.4 & 2.7 & 15.99 & 5.49 \\
\hline Chihuahua & 2.7 & 5.4 & 0.6 & 9.04 & 6.39 \\
\hline Distrito Federal & 0.7 & 3.6 & 12.7 & 6.21 & 17.46 \\
\hline Durango & 3.0 & 7.3 & 0.2 & 9.83 & 6.09 \\
\hline Guanajuato & 1.6 & 4.0 & 2.7 & 14.02 & 5.18 \\
\hline Guerrero & 2.8 & 4.7 & 1.3 & 12.10 & 6.85 \\
\hline Hidalgo & 1.4 & 12.8 & 6.3 & 17.99 & 9.37 \\
\hline Jalisco & 0.7 & 3.6 & 5.1 & 9.85 & 8.15 \\
\hline México & 0.5 & 4.8 & 2.3 & 9.51 & 10.95 \\
\hline Michoacán & 2.0 & 4.1 & 9.1 & 17.78 & 8.75 \\
\hline Morelos & 1.6 & 4.2 & 10.8 & 10.08 & 8.03 \\
\hline $\mathrm{N}$ ayarit & 1.7 & 4.5 & 2.3 & 12.32 & 10.18 \\
\hline Nuevo León & 1.0 & 9.2 & 1.2 & 8.43 & 4.15 \\
\hline 0 axaca & 5.1 & 4.9 & 4.8 & 23.48 & 6.04 \\
\hline Puebla & 4.0 & 5.0 & 4.2 & 18.30 & 5.31 \\
\hline Q uerétaro & 1.8 & 4.9 & 3.1 & 13.57 & 9.31 \\
\hline Q uintana Roo & 2.1 & 3.6 & 10.1 & 10.39 & 8.56 \\
\hline San Luis Potosí & 3.1 & 6.9 & 2.0 & 14.10 & 6.34 \\
\hline Sinaloa & 1.9 & 5.5 & 2.4 & 7.51 & 10.28 \\
\hline Sonora & 1.5 & 4.9 & 0.6 & 8.77 & 5.36 \\
\hline Tabasco & 1.5 & 3.1 & 10.4 & 10.66 & 7.67 \\
\hline Tamaulipas & 1.3 & 4.4 & 6.3 & 9.06 & 8.59 \\
\hline Tlaxcala & 2.6 & 4.3 & 6.1 & 18.99 & 5.68 \\
\hline Veracruz & 1.9 & 2.5 & 20.0 & 13.98 & 4.83 \\
\hline Yucatán & 4.7 & 2.8 & 4.2 & 9.73 & 4.54 \\
\hline Zacatecas & 2.5 & 5.4 & 0.6 & 13.26 & 7.35 \\
\hline
\end{tabular}

* Porcentaje respecto al total de defunciones

₹ Porcentaje respecto al total de tumores malignos C00-C $97 *$

$\S$ Porcentaje respecto al total de defunciones por causa externaV01-Y98*

* Porcentaje respecto a las enfermedades del corazón 100-151*

\& Porcentaje respecto a las enfermedades del hígado K $70^{\prime} \mathrm{K} 76^{*}$

* Códigos de la CIE 10: C lasificación Estadística Internacional de Enfermedades y Problemas Relacionados con la Salud. 10a revisión

Fuente: Instituto Nacional de Estadística, Geografía e Informática/Secretaría de Salud. Dirección General de Información en Salud. Base de datos de defunciones 2002. México, 2004 


\section{Cuadro XV \\ MorTalidad POR Ciertas afecciones Perinatales (P00-P96,A33), * SEgún ENTIDAd federativa de Residencia habitual. México, 2000-2002}

\begin{tabular}{|c|c|c|c|c|c|c|c|c|c|c|c|c|}
\hline \multirow[b]{3}{*}{ Entidad federativa } & \multicolumn{4}{|c|}{2000} & \multicolumn{4}{|c|}{2001} & \multicolumn{4}{|c|}{2002} \\
\hline & \multicolumn{2}{|c|}{ M ujeres } & \multicolumn{2}{|c|}{ Hombres } & \multicolumn{2}{|c|}{ M ujeres } & \multicolumn{2}{|c|}{ Hombres } & \multicolumn{2}{|c|}{ M ujeres } & \multicolumn{2}{|c|}{ Hombres } \\
\hline & Def & Tasa & Def & Tasa & Def & Tasa & Def & Tasa & Def & Tasa & Def & Tasa \\
\hline$N$ acional & 8107 & 787.6 & 11260 & 1046.9 & 7693 & 764.9 & 10452 & 994.4 & 7810 & 790.5 & 10716 & 1037.6 \\
\hline A guascalientes & 84 & 761.7 & 126 & 1093.8 & 93 & 860.2 & 111 & 983.2 & 86 & 811.2 & 126 & 1138.0 \\
\hline Baja California & 226 & 834.1 & 297 & 1047.8 & 177 & 657.7 & 292 & 1036.0 & 194 & 728.7 & 286 & 1025.5 \\
\hline Baja California Sur & 33 & 746.3 & 41 & 885.1 & 26 & 589.4 & 60 & 1295.6 & 34 & 771.9 & 50 & 1081.1 \\
\hline Campeche & 50 & 640.9 & 56 & 686.7 & 47 & 613.4 & 54 & 674.0 & 43 & 571.7 & 57 & 724.5 \\
\hline Coahuila & 119 & 489.1 & 146 & 573.8 & 116 & 487.0 & 168 & 673.8 & 105 & 448.8 & 176 & 718.6 \\
\hline Colima & 37 & 714.4 & 52 & 961.2 & 31 & 605.6 & 45 & 841.8 & 25 & 490.8 & 34 & 639.1 \\
\hline Chiapas & 318 & 653.3 & 463 & 910.9 & 340 & 716.7 & 483 & 975.4 & 327 & 704.2 & 459 & 946.8 \\
\hline Chihuahua & 267 & 825.8 & 392 & 1159.1 & 277 & 870.7 & 396 & 1188.8 & 311 & 990.7 & 415 & 1262.5 \\
\hline Distrito Federal & 663 & 940.5 & 933 & 1267.5 & 635 & 918.6 & 870 & 1206.1 & 660 & 959.4 & 817 & 1138.0 \\
\hline Durango & 22 & 134.9 & 31 & 182.0 & 28 & 177.7 & 28 & 170.2 & 76 & 498.3 & 150 & 941.5 \\
\hline Guanajuato & 526 & 949.5 & 755 & 1304.1 & 468 & 875.7 & 619 & 1108.0 & 455 & 881.8 & 683 & 1266.0 \\
\hline Guerrero & 136 & 357.1 & 199 & 500.2 & 108 & 294.4 & 148 & 386.1 & 118 & 332.7 & 193 & 520.7 \\
\hline Hidalgo & 179 & 763.7 & 229 & 935.3 & 172 & 753.5 & 206 & 863.9 & 174 & 775.7 & 266 & 1135.1 \\
\hline Jalisco & 460 & 679.8 & 674 & 953.1 & 462 & 701.7 & 677 & 983.4 & 459 & 714.0 & 605 & 900.0 \\
\hline México & 1448 & 1086.5 & 2057 & 1476.9 & 1404 & 1066.0 & 1929 & 1401.2 & 1399 & 1066.0 & 1780 & 1297.4 \\
\hline Michoacán & 233 & 530.8 & 368 & 802.4 & 234 & 553.4 & 361 & 817.0 & 228 & 556.4 & 361 & 842.9 \\
\hline Morelos & 110 & 692.2 & 141 & 849.2 & 107 & 685.1 & 130 & 796.7 & 118 & 765.0 & 157 & 974.1 \\
\hline $\mathrm{N}$ ayarit & 37 & 385.5 & 52 & 518.3 & 30 & 320.3 & 47 & 479.7 & 37 & 401.4 & 43 & 446.0 \\
\hline N uevo León & 207 & 535.1 & 301 & 744.2 & 200 & 525.7 & 264 & 663.4 & 203 & 541.3 & 275 & 701.0 \\
\hline 0 axaca & 286 & 731.0 & 390 & 954.4 & 242 & 636.7 & 318 & 801.1 & 300 & 806.2 & 429 & 1103.8 \\
\hline Puebla & 816 & 1459.6 & 1074 & 1839.1 & 740 & 1358.2 & 995 & 1748.2 & 704 & 1316.7 & 1043 & 1867.1 \\
\hline Q uerétaro & 138 & 884.6 & 170 & 1043.2 & 134 & 872.8 & 180 & 1122.5 & 147 & 969.5 & 165 & 1041.7 \\
\hline Q uintana Roo & 88 & 872.9 & 94 & 891.8 & 77 & 758.8 & 112 & 1055.1 & 75 & 732.1 & 111 & 1035.6 \\
\hline San Luis Potosí & 173 & 678.5 & 242 & 908.3 & 146 & 600.0 & 210 & 825.8 & 159 & 690.0 & 229 & 950.7 \\
\hline Sinaloa & 58 & 217.5 & 78 & 279.8 & 46 & 177.2 & 56 & 206.2 & 81 & 319.7 & 111 & 418.8 \\
\hline Sonora & 194 & 824.3 & 244 & 991.5 & 166 & 717.3 & 202 & 834.1 & 170 & 746.2 & 225 & 943.6 \\
\hline Tabasco & 158 & 783.1 & 284 & 1347.3 & 180 & 909.0 & 235 & 1135.7 & 168 & 856.2 & 199 & 970.4 \\
\hline Tamaulipas & 186 & 645.9 & 202 & 671.0 & 156 & 548.3 & 184 & 618.4 & 139 & 492.3 & 148 & 501.2 \\
\hline Tlaxcala & 112 & 1097.5 & 166 & 1557.7 & 115 & 1141.1 & 155 & 1473.4 & 101 & 1007.8 & 163 & 1557.9 \\
\hline Veracruz & 517 & 753.7 & 694 & 968.3 & 491 & 738.6 & 615 & 885.4 & 478 & 736.8 & 676 & 997.1 \\
\hline Yucatán & 109 & 669.2 & 162 & 951.7 & 131 & 813.9 & 171 & 1016.2 & 107 & 667.4 & 126 & 751.7 \\
\hline Zacatecas & 117 & 767.8 & 147 & 923.9 & 114 & 775.4 & 131 & 853.8 & 129 & 902.2 & 158 & 1058.6 \\
\hline
\end{tabular}

* Códigos de la CIE 10:Clasificación Estadística Internacional de Enfermedades y Problemas Relacionados con la Salud. 10a revisión

Tasa por 100000 habitantes menores de un año (no estandarizada)

Los totales no incluyen defunciones de mujeres y hombres residentes en el extranjero

Fuente: Instituto Nacional de Estadística, Geografía e Informática/Secretaría de Salud. Dirección General de Información en Salud. Base de datos de defunciones 2002. México, 2004

Consejo N acional de Población. Proyecciones de la Población de México, 2000-2050. México, 2002 